\title{
Solution of the Quintic and Sextic by Radicals
}

\author{
Jorge A. Franco ${ }^{1 *}$
}

1 Independent researcher. https://orcid.org/ 0000-0001-5951-1129. georgeafr@gmail.com.

* Correspondence: georgeafr@gmail.com, jafrancor@yahoo.com, journal.of.vr@hotmail.com

\begin{abstract}
Applying a procedure similar to that of E.S. Bring, by using a $4^{\text {th }}$ degree Tschirnhaus transformation, it was possible to transform the Bring-Jerrard normal quintic (BJQ) equation into a De Moivre form (DMQ), so that it could be solved by radicals. The general solution by radicals of the De Moivre equations of any degree is presented. By the same procedure the BJSx (normal sextic) equation was taken to another one without the $2^{\text {nd }}, 4^{\text {th }}$ and $6^{\text {th }}$ terms which was transformed into a cubic (solvable) equation. By applying a $6^{\text {th }}$ degree Tschirnhaus transformation to the BJSp (normal septic) equation its binormal (without the $2^{\text {nd }}, 3^{\text {rd }}, 4^{\text {th }}$ and $5^{\text {th }}$ terms) form was obtained.
\end{abstract}

Keywords: Solution of the Quintic by Radicals; Solution of the Sextic by Radicals; Solution of the De Moivre Equation of any degree by Radicals; Eliminating Four Terms at once from the Septic.

\section{Introduction}

So far there have been many unsuccessful attempts to solve the general quintic equation by radicals by mathematicians of all times: E. Tschirnhaus (1651-1708), A. De Moivre (1667-1754), E.S. Bring (1736-1798), L. Euler (1707-1783), G. F. Malfatti (17311807), E.B. Jerrard (1804-1863), G. N. Watson (1886-1965), E. S. Dummit (paper, 1991) and many others. However, since Paolo Ruffini (1765-1822), Niels Henrik Abel (1802-1829) and Évariste Galois (1811-1822) wrote about proofs that the general quintic is not solvable by radicals, these attempts, have been greatly reduced. The meaning of the phrase by radicals refers to the roots of such equations as functions of the coefficients, obtained them by means of a finite number of algebraic operations. The main objective of this paper is to present an approach to solve quintics by radicals, following Bring's procedure.

In this work we develop the foundations for obtaining the general formulae for solving the De Moivre equations in 2.2. The method used to solve the quintic is described in 2.3. Applications of the method to reduce general equations of degrees 3, 4, 5 to the De Moivre form and solve them by radicals are shown in sections 3.1, 3.2, and 3.3. Sextic is solved in section 3.4. Septic is treated in section 3.5 and four terms were eliminated at once from this equation. Comments and Conclusions are presented in section 4 .

\section{Foundations and Methods}

As is known, a general quintic monic equation $f(z)=z_{5}+\sum_{1}^{5} Z_{5-j} z^{5-j}=0$, can be reduced by sequential Tschirnhaus transformations of the $1^{\text {st }}, 2^{\text {nd }}$ and $4^{\text {th }}$ degree, to the Bring-Jerrard normal form, $x^{5}+q x+r=0$. For achieving this, E. S. Bring, in 1786 used a $4^{\text {th }}$ degree transformation whose coefficients were: $c=d+\gamma, b=\sigma d+\zeta$ and $a=$ $(3 p d+4 q) / 5[1-3]$, in order to avoid degrees of the auxiliary equations greater than 4 .

On the other hand, when it is possible to eliminate inter-sequential terms, by any transformation that allows it, of a general monic equation, $z^{n}+\sum_{1}^{n} Z_{n-j} z^{n-j}=0$, it results:

For odd $n$ :

For even $n$ :

$$
\begin{gathered}
y^{n}+\sum_{p=1}^{\frac{n-1}{2}} Y_{n-2 p} y^{n-2 p}+Y_{0}=0 ; \\
y^{n}+\sum_{p=1}^{n / 2} Y_{n-2 p} y^{n-2 p}=0
\end{gathered}
$$


and, if the coefficients can respond as: $Y_{n-2 p}=(-1)^{p} C_{n-2 p}\left(\frac{Y_{n-2}}{C_{n-2}}\right)^{p}=(-1)^{p} C_{n-2 p} \alpha^{p}$, for $\alpha=\frac{Y_{n-2}}{C_{n-2}}$, the De Moivre equation of odd or even degree, (2) and (3) below, arises:

$$
\begin{aligned}
& \left\{\begin{array}{l}
\text { For } n \text { odd: } y^{n}+\sum_{p=1}^{\frac{n-1}{2}}(-1)^{p} C_{n-2 p} \alpha^{p} y^{n-2 p}-C_{0}=0 \\
\text { For } n \text { even: } y^{n}+\sum_{p=1}^{\frac{n}{2}-1}(-1)^{p} C_{n-2 p} \alpha^{p} y^{n-2 p}-C_{0}=0
\end{array}\right\}, \text { or } \\
& y^{n}-C_{n-2} \alpha y^{n-2}+\cdots+C_{n-2 p} \alpha^{p} y^{n-2 p}-\cdots \pm C_{n-2\left(\frac{n-1}{2}\right)} \alpha^{\frac{n-1}{2}} y-C_{0}=0 \\
& y^{n}-C_{n-2} \alpha y^{n-2}+\cdots+C_{n-2 p} \alpha^{p} y^{n-2 p}-\cdots \pm C_{n-2\left(\frac{n-2}{2}\right)} \alpha^{\frac{n-2}{2}} y^{2}-C_{0}=0
\end{aligned}
$$

As will be shown in 2.1, any De Moivre's equation can be solved by radicals.

\subsection{Foundations.}

In general, a monic equation of degree $n, z^{n}+\sum_{1}^{n} Z_{n-j} z^{n-j}=0$, reduced to the De Moivre form (2) and (3) can be compared with the expansion of $(u+v)^{n}$, converted into a De Moivre equation. Such expansion can be represented either by the well-known power of a binomial, or by an identity which has the natural De Moivre structure, i.e.:

$$
\begin{gathered}
(u+v)^{n} \equiv\left(\begin{array}{c}
n \\
0
\end{array}\right) u^{n}+\left(\begin{array}{c}
n \\
1
\end{array}\right) v u^{n-1}+\cdots+\left(\begin{array}{c}
n \\
n-1
\end{array}\right) v^{n-1} u+\left(\begin{array}{l}
n \\
n
\end{array}\right) v^{n} \\
(u+v)^{n} \equiv C_{n-2}(u v)(u+v)^{n-2}-C_{n-4}(u v)^{2}(u+v)^{n-4}+\cdots+C_{0}
\end{gathered}
$$

The last identity is constructed by using the sum of symmetric terms within the first identity and completing the factors $(u+v)^{i}$ with the missing elements. Then, passing all terms to the left-hand side of the equal sign, we convert the identity into an equation. For example:

$$
\begin{aligned}
& (p+q)^{3}=p^{3}+3 p^{2} q+3 p q^{2}+q^{3}=3 p q(p+q)+p^{3}+q^{3} \rightarrow(\boldsymbol{p}+\boldsymbol{q})^{\mathbf{3}}-\mathbf{3 p q}(\boldsymbol{p}+\boldsymbol{q})-\left(\boldsymbol{p}^{\mathbf{3}}+\boldsymbol{q}^{\mathbf{3}}\right)=\mathbf{0} \\
& (p+q)^{4}=p^{4}+4 p^{3} q+6 p^{2} q^{2}+4 p q^{3}+q^{4}=4 p q\left(p^{2}+q^{2}\right)+6 p^{2} q^{2}+p^{4}+q^{4}+[4 p q(2 p q)-4 p q(2 p q)] \\
& =4 p q(p+q)^{2}+p^{4}+q^{4}-2 p^{2} q^{2} \rightarrow(\boldsymbol{p}+\boldsymbol{q})^{\mathbf{4}}-\mathbf{4} \boldsymbol{p q}(\boldsymbol{p}+\boldsymbol{q})^{2}-\left(\boldsymbol{p}^{2}-\boldsymbol{q}^{2}\right)^{\mathbf{2}}=\mathbf{0} \\
& (p+q)^{5}=p^{5}+5 p^{4} q+10 p^{3} q^{2}+10 p^{2} q^{3}+5 p q^{4}+q^{5} \\
& =5 p q\left(p^{3}+q^{3}\right)+10 p^{2} q^{2}(p+q)+p^{5}+q^{5}+\left[5 p q\left(3 p^{2} q+3 p q^{2}\right)-5 p q\left(3 p^{2} q+3 p q^{2}\right)\right] \\
& =5 p q(p+q)^{3}-5 p^{2} q^{2}(p+q)+p^{5}+q^{5} \rightarrow(\boldsymbol{p}+\boldsymbol{q})^{\mathbf{5}}-\mathbf{5} \boldsymbol{p} \boldsymbol{q}(\boldsymbol{p}+\boldsymbol{q})^{\mathbf{3}}+\mathbf{5} \boldsymbol{p}^{\mathbf{2}} \boldsymbol{q}^{\mathbf{2}}(\boldsymbol{p}+\boldsymbol{q})-\left(\boldsymbol{p}^{\mathbf{5}}+\boldsymbol{q}^{\mathbf{5}}\right)=\mathbf{0} \\
& (p+q)^{6}=p^{6}+6 p^{5} q+15 p^{4} q^{2}+20 p^{3} q^{3}+15 p^{2} q^{4}+6 p q^{5}+q^{6}=6 p q\left(p^{4}+q^{4}\right)+15 p^{2} q^{2}\left(p^{2}+q^{2}\right)+20 p^{3} q^{3}+p^{6}+q^{6} \\
& +\left[6 p q\left(4 p^{3} q+6 p^{2} q^{2}+4 p q^{3}\right)-6 p q\left(4 p^{3} q+6 p^{2} q^{2}+4 p q^{3}\right)\right]+\left[15 p^{2} q^{2}(2 u v)-15 p^{2} q^{2}(2 u v)\right] \\
& +\left[2 p^{3} q^{3}-2 p^{3} q^{3}\right] \quad \rightarrow \quad(\boldsymbol{p}+\boldsymbol{q})^{\mathbf{6}}-\mathbf{6} \boldsymbol{p} \boldsymbol{q}(\boldsymbol{p}+\boldsymbol{q})^{\mathbf{4}}+\mathbf{9} \boldsymbol{p}^{\mathbf{2}} \boldsymbol{q}^{\mathbf{2}}(\boldsymbol{p}+\boldsymbol{q})^{\mathbf{2}}-\left(\boldsymbol{p}^{3}+\boldsymbol{q}^{3}\right)^{2}=\mathbf{0}
\end{aligned}
$$

This feature of $(u+v)^{n}$, of naturally becoming the De Moivre structure, see eq. (4) below, allows us to take it as a model with which to compare equations (2), (3), and solve them using $y=u+v, \alpha=u v, C_{n-2}=n$ and $C_{0}=\beta_{n}$.

$$
(u+v)^{n}-n(u v)(u+v)^{n-2}+\cdots \mp C_{n-2 p}(u v)^{p}(u+v)^{n-2 p} \pm \cdots-\beta_{n}=0
$$

Moreover, and even better, the coefficients $C_{n-2 p}$ of equation (4), $n$ even or odd, can be structured as a right triangle, with $n$ rows and $p$ columns $(p=0,1, \ldots)$, see Table 1 , where the one-dimensional coefficients $\boldsymbol{C}_{\boldsymbol{n}-2 \boldsymbol{p}}$ in eq. (4), when placed in the right triangle, change to a two-dimensional expression $\boldsymbol{C}_{\boldsymbol{n}, \boldsymbol{p}}$. In this way, the coefficients $C_{n-2 p}$ can be calculated as diagonal sums in a style similar to that of Pascal's or Tartaglia's triangle. The factors $\alpha^{p}$ in the terms of (4), and the $\beta_{n}$, are also easily obtained. The following properties of the terms can be visualized as: 
- $\quad$ For odd $n \geq 5$, absolute values of extreme internal coefficients in (2) are:

$$
\left|C_{n-2}\right|=\left|C_{n-2 \frac{(n-1)}{2}}\right|=n
$$

The same definition, expressed in two dimensions, in Table 1 below, becomes:

$$
\left|C_{n, 1}\right|=\left|C_{n, \frac{(n-1)}{2}}\right|=n .
$$

- $\quad$ Filling the far right inside the even $n$ lines with the value 2, or -2, helps us to build all the results down. But, such numbers are not real coefficients! They are just filler numbers, to construct diagonal sums (eq. (6) below) in table 1.

- For even $n \geq 4$, the last term occurs for $p=n / 2$ and for $n \geq 5$ for $p=(n-1) / 2$.

- Results of diagonal sums (including the filler numbers 2 or -2 ) respond to:

$$
\left|C_{n, p}\right|=(-1)^{p}\left(\left|C_{(n-2),(p-1)}\right|+\left|C_{(n-1), p}\right|\right)
$$

- If $n$ is odd, $\beta_{n}=u^{n}+v^{n}$. But, if $n$ is even, $\beta_{n}=\left(u^{n / 2} \pm v^{n / 2}\right)^{2}$, and sign \pm inside $\beta_{n}$ is given by, $\pm=(-1)^{(n / 2-1)}$. Thus, if we consider $n$ even and $n / 2$ odd, sign within $\beta_{n}$, is + ; but, if $n / 2$ is even, sign is - . Check this in Table 1:

\begin{tabular}{|l|l|l|l|l|l|l|l|c|c|}
\hline$n \downarrow p \rightarrow$ & 0 & 1 & 2 & 3 & 4 & 5 & 6 & $\ldots$ & $\beta_{\mathbf{n}}$ \\
\hline 1 & 1 & & & & & & & & $-(u+v)$ \\
\hline 2 & 1 & -2 & & & & & & & $-\left(u^{2 / 2}+v^{2 / 2}\right)^{2}$ \\
\hline 3 & 1 & -3 & & & & & & & $-\left(u^{3}+v^{3}\right)$ \\
\hline 4 & 1 & -4 & 2 & & & & & & $-\left(u^{4 / 2}-v^{4 / 2}\right)^{2}$ \\
\hline 5 & 1 & -5 & 5 & & & & & & $-\left(v^{5}+v^{5}\right)$ \\
\hline 6 & 1 & -6 & 9 & -2 & & & & & $-\left(u^{6 / 2}+v^{6 / 2}\right)^{2}$ \\
\hline 7 & 1 & -7 & 14 & -7 & & & & & $-\left(u^{7}+v^{7}\right)$ \\
\hline 8 & 1 & -8 & 20 & -16 & 2 & & & & $-\left(u^{8 / 2}-v^{8 / 2}\right)^{2}$ \\
\hline 9 & 1 & -9 & 27 & -30 & 9 & & & & $-\left(u^{9}+v^{9}\right)$ \\
\hline 10 & 1 & -10 & 35 & -50 & 25 & -2 & & & $-\left(u^{10 / 2}+v^{10 / 2}\right)^{2}$ \\
\hline 11 & 1 & -11 & 44 & -77 & 55 & -11 & & & $-\left(u^{11}+v^{11}\right)$ \\
\hline 12 & 1 & -12 & 54 & -112 & 105 & -36 & 2 & & $-\left(u^{12 / 2}-v^{12 / 2}\right)^{2}$ \\
\hline 13 & 1 & -13 & 65 & -156 & 182 & -91 & $\mathbb{1 3}$ & & $-\left(u^{13}+v^{13}\right)$ \\
\hline
\end{tabular}

Table 1. Rectangular triangle: the result of diagonal sums of the coefficients is displayed vertically downwards. This rule allows us calculate all the coefficients.

\subsection{Solving the general De Moivre Equation by radicals.}

Next is obtained the general solution, by radicals, of the De Moivre equation, where, as will be seen, it depends only on $\alpha$ or $Y_{n-2}$, and $\beta_{n}$ or $Y_{0}$. To do so, let us first compare the equation of degree $n$ odd (7), in De Moivre's form, with (8), below

$$
\begin{gathered}
y^{n}-Y_{n-2} y^{n-2}+Y_{n-4} y^{n-4}-Y_{n-6} y^{n-6}+\cdots \pm Y_{1} y-Y_{0}=0 \\
y^{n}-C_{n-2} \alpha y^{n-2}+C_{n-4} \alpha^{2} y^{n-4}-C_{n-6} \alpha^{3} y^{n-6} \mp \cdots \pm C_{n-2\left(\frac{n-1}{2}\right)^{\frac{n-1}{2}} y-C_{0}=0} \\
(u+v)^{n}-n(u v)(u+v)^{n-2}+\cdots \mp C_{n-2 p}(u v)^{p}(u+v)^{n-2 p} \pm \ldots \pm C_{n-2\left(\frac{n-1}{2}\right)} \alpha^{\frac{n-1}{2}} y-\beta_{n}=0
\end{gathered}
$$

Where, for $y=u+v, Y_{n-2}=C_{n-2} \alpha=n(u v)$ and $Y_{1}=C_{1} \alpha^{\frac{n-1}{2}}=n \alpha^{\frac{n-1}{2}}$ :

$$
\begin{aligned}
& Y_{n-2}=n \alpha \quad ; \quad u=\frac{Y_{n-2}}{n v}, \quad ; \quad Y_{n-2 p}=c_{n-2 p} \alpha^{p}=c_{n-2 p}\left(\frac{Y_{n-2}}{n}\right)^{p} \\
& \alpha=\frac{Y_{n-2}}{n}=u v ; \quad v=\frac{Y_{n-2}}{n u} \quad ; \quad r \begin{aligned}
Y_{0} & =C_{0}=\beta_{n}=u^{n}+v^{n}
\end{aligned}
\end{aligned}
$$


These relationships give rise to auxiliary equations and their solutions:

$$
\begin{gathered}
u^{2 n}-Y_{0} u^{n}+\left(\frac{Y_{n-2}}{n}\right)^{n}=0 \rightarrow u^{n}=\frac{Y_{0}}{2}+\frac{1}{2} \sqrt{Y_{0}^{2}-4\left(\frac{Y_{n-2}}{n}\right)^{n}} \rightarrow u=\sqrt[n]{\frac{Y_{0}}{2}+\frac{1}{2} \sqrt{Y_{0}^{2}-4\left(\frac{Y_{n-2}}{n}\right)^{n}}} \\
v^{n}=Y_{0}-u^{n} \rightarrow v^{n}=\frac{Y_{0}}{2}-\frac{1}{2} \sqrt{Y_{0}^{2}-4\left(\frac{Y_{n-2}}{n}\right)^{n}} \rightarrow v=\sqrt[n]{\frac{Y_{0}}{2}-\frac{1}{2} \sqrt{Y_{0}^{2}-4\left(\frac{Y_{n-2}}{n}\right)^{n}}}
\end{gathered}
$$

A basic solution immediately appears, $y_{1}=u+v$. The solution for odd $\boldsymbol{n}$ arises by multiplying $u$ and $v$ by the unit roots, $\omega_{m}=e^{j\left(m \frac{2 \pi}{n}\right)}$, giving exactly the necessary $n$ roots, by using $y_{k}=\omega_{k-1} u+\omega_{n-(k-1)} v$, as follows:

$$
\begin{aligned}
& y_{k}=\omega_{k-1} \sqrt[n]{\frac{Y_{0}}{2}+\frac{1}{2} \sqrt{Y_{0}^{2}-4\left(\frac{Y_{n-2}}{n}\right)^{n}}}+\omega_{n-(k-1)} \sqrt[n]{\frac{Y_{0}}{2}-\frac{1}{2} \sqrt{Y_{0}^{2}-4\left(\frac{Y_{n-2}}{n}\right)^{n}}} \\
& y_{1}=u+v=\omega_{0} u+\omega_{n} v
\end{aligned}
$$

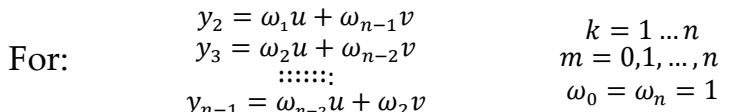

$$
\begin{aligned}
& y_{n}=\omega_{n-1} u+\omega_{1} v
\end{aligned}
$$

Now, comparing the equation of even degree $\boldsymbol{n}$, in (1) or (11), and the De Moivre's form (3), with the binomial form (12), below:

$$
\begin{aligned}
& y^{n}-Y_{n-2} y^{n-2}+Y_{n-4} y^{n-4}-\cdots \pm Y_{n-2 p} y^{n-2 p} \mp \cdots \pm Y_{2} y^{2}-Y_{0}=0 \\
& y^{n}-C_{n-2} \alpha y^{n-2}+\cdots \pm C_{n-2 p} \alpha^{p} y^{n-2 p}-\cdots \pm C_{n-2\left(\frac{n-2}{2}\right)^{\frac{(n-2)}{2}} y^{2}-C_{0}=0} \\
& (u+v)^{n}-n(u v)(u+v)^{n-2}+\cdots \pm C_{n-2 \frac{n-2}{2}}(u v)^{\frac{n-2}{2}}(u+v)^{n-2 \frac{n-2}{2}}-\beta_{n}=0
\end{aligned}
$$

Where: for $v=\frac{Y_{n-2}}{n u}, u=\frac{Y_{n-2}}{n v}$, and $Y_{0}=C_{0}=\beta_{n}=\left(u^{n / 2} \pm v^{n / 2}\right)^{2}$, two auxiliary equations, become created:

$$
\begin{aligned}
& C_{n-2}=n ; \quad Y_{n-2}=n \alpha ; \quad \alpha=u v ; Y_{n-2 p}=C_{n-2 p} \alpha^{p} ; \quad Y_{0}=\beta_{n}=\left(u^{n / 2} \pm v^{n / 2}\right)^{2} \\
& u^{n}-\sqrt{Y_{0}} u^{\frac{n}{2}} \mp\left(\frac{Y_{n-2}}{n}\right)^{\frac{n}{2}}=0 \rightarrow u=\sqrt[n]{\frac{\sqrt{Y_{0}}}{2}+\frac{1}{2} \sqrt{Y_{0} \pm 4\left(\frac{Y_{n-2}}{n}\right)^{\frac{n}{2}}}} \\
& v^{\frac{n}{2}}=Y_{0}-u^{\frac{n}{2}} \rightarrow v^{\frac{n}{2}}=\frac{\sqrt{Y_{0}}}{2}-\frac{1}{2} \sqrt{Y_{0} \pm 4\left(\frac{Y_{n-2}}{n}\right)^{\frac{n}{2}}} \rightarrow v=\sqrt[n]{\frac{\sqrt[n]{Y_{0}}}{2}-\frac{1}{2} \sqrt{Y_{0} \pm 4\left(\frac{Y_{n-2}}{n}\right)^{\frac{n}{2}}}}
\end{aligned}
$$

The basic solution, $y_{1}=u+v=\omega_{0} u+\omega_{n} v$, and the others (with the same definitions), $y_{k}=\omega_{k-1} u+\omega_{n-(k-1)} v$, for even $\boldsymbol{n}$, are:

$$
y_{k}=\omega_{k-1} \sqrt[\frac{n}{2}]{\frac{\sqrt{Y_{0}}}{2}+\frac{1}{2} \sqrt{Y_{0} \pm 4\left(\frac{Y_{n-2}}{n}\right)^{\frac{n}{2}}}}+\omega_{n-(k-1)} \sqrt[\frac{n}{2}]{\frac{\sqrt{Y_{0}}}{2}-\frac{1}{2} \sqrt{Y_{0} \pm 4\left(\frac{Y_{n-2}}{n}\right)^{\frac{n}{2}}}}
$$




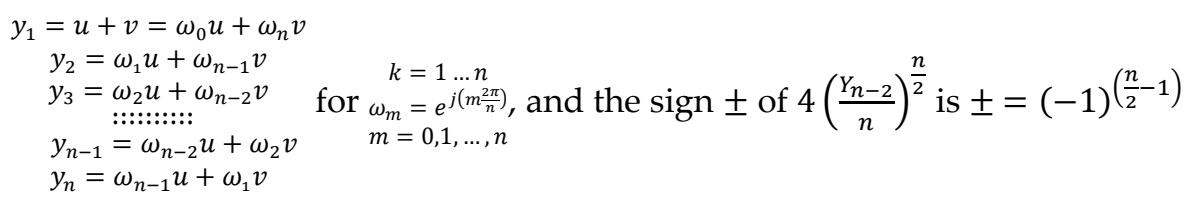

\subsection{Reducing any monic quintic equation to the De Moivre form.}

1. First, apply a $1^{\text {st }}$ degree Tschirnhaus transformation (TschT) to the general monic quintic to obtain its reduced form without the 2 nd term, $z_{5}+\sum_{2}^{5} Z_{5-j} z^{5-j}=0$; then a $2^{\text {nd }}$ degree TschT to the reduced equation to obtain the principal form without the $1^{\text {st }}$ and $2^{\text {nd }}$ term, $y_{5}+\sum_{3}^{5} Y_{5-j} y^{5-j}=0$; and, apply the Bring's expression of the $4^{\text {th }}$ degree TschT, $y^{4}+d y^{3}+c y^{2}+b y+a-x=0$ to the principal, with coefficients: $c=d+\gamma$, $\boldsymbol{b}=\sigma \boldsymbol{d}+\zeta$, and $\boldsymbol{a}=(3 p \boldsymbol{d}+4 q) / 5$, to obtain the normal form, $x^{5}+q x+r=0$, also called Bring-Jerrard normal form (BJQ). The BJQ is required to follow with the next point 2.

2. Apply another TschT of the $4^{\text {th }}$ degree, $x^{4}+d x^{3}+c x^{2}+b x+a-w=0$, by using the following expressions for the coefficients, $\boldsymbol{c}=\boldsymbol{d}+h+i, \boldsymbol{b}=f \boldsymbol{d}+h+j$ and $\boldsymbol{a}=4 q / 5$, to the BJQ $\left(x^{5}+q x+r=0\right)$, to obtain the De Moivre Quintic (DMQ), $w^{5}-W_{3} \alpha w^{3}+$ $W_{1} \alpha^{2} w-W_{0}=0$. Notice that by using this new $4^{\text {th }}$ degree TschT, with different coefficients than those used by Bring in the previous 4th degree TschT, we were able to obtain the De Moivre form from the BJQ with auxiliary equations for $n<5$ degree in a simple way.

3. Solve the obtained DMQ equation by comparing it with the quintic binomial power, $(u+v)^{5}-5 u v(u+v)^{3}+5 u^{2} v^{2}(u+v)-\left(u^{5}+v^{5}\right)=0$.

4. And finally, reverse all the transformations and changes made, until returning to the initial equation to determine its original roots. To undo them, it was necessary to obtain the inverse formulas that solved both the Tschirnhaus transformations used and all necessary changes.

5. Something like this was also applied to the sextic equation. To become familiar with this method, it is applied in a simple way to equations of degrees 3 and 4 , and then its (longer and more laborious) application to the quintic equation is developed.

\section{Results}

\subsection{Solving the Cubic Equation}

A first degree Tschirnhaus transformation, $y=x+\frac{b}{3^{\prime}}$ is applied to the general monic equation of the $3^{\text {rd }}$ degree to eliminate the $2^{\text {nd }}$ term. This transforms the resultant directly into the De Moivre cubic structure. A flowchart summarizing this calculation is:

$$
\begin{array}{lll}
f(x)=x^{3}+b x^{2}+c x+d=0 \downarrow & \leftarrow & x=-\frac{b}{3}+y \\
\stackrel{\downarrow}{\rightarrow} \& g(x)=y-\left(x+\frac{b}{3}\right)=0 \rightarrow \operatorname{Res}[f(x), g(x)]=y^{3}-Y_{1} y-Y_{0}=0 \rightarrow y_{1,2,3} \uparrow
\end{array}
$$

Figure 1. Flowchart showing the steps to solve the Cubic Equation

The expressions of $Y_{1}$ and $Y_{0}$, calculated through the Sylvester matrix become:

$$
\left\{\begin{array}{c}
Y_{1}=-\left(c-\frac{1}{3} b^{2}\right) \\
Y_{0}=-\left(\frac{2}{27} b^{3}-\frac{1}{3} b c+d\right)
\end{array}\right\}
$$




$$
x_{k}=-\frac{b}{3}+\omega_{k-1} \sqrt[3]{\frac{Y_{0}+\sqrt{Y_{0}^{2}-\left(\frac{Y_{1}}{3}\right)^{3}}}{2}+\omega_{n-(k-1)} \sqrt[3]{\frac{Y_{0}-\sqrt{Y_{0}^{2}-4\left(\frac{Y_{1}}{3}\right)^{3}}}{2}}} \begin{aligned}
& x_{1}=-\frac{b}{3}+\omega_{0} u+\omega_{3} v \\
& x_{2}=-\frac{b}{3}+\omega_{1} u+\omega_{2} v \\
& x_{3}=-\frac{b}{3}+\omega_{2} u+\omega_{1} v
\end{aligned}
$$

As seen, by using the solution for $y_{k}$, odd $n$ in (10), and undoing the change to $x=$ $-\frac{b}{3}+y$, we obtain a formula similar to Cardano's [5]:3

\subsection{Solving the Quartic Equation}

Starting from a reduced quartic without the $2^{\text {nd }}$ term (to simplify, not required), $f(x)=x^{4}+c x^{2}+d x+e=0$, we apply a $2^{\text {nd }}$ degree Tschirnhaus transformation, $g(x)=$ $x^{2}+p x+q-y$, in order to eliminate the $2^{\text {nd }}$ and $4^{\text {th }}$ terms from the resultant and obtain its De Moivre form (DMF), whose general solution is directly given by equation (13):

$$
\begin{array}{lrr}
f(x)=x^{4}+c x^{2}+d x+e=0 & \leftarrow & x_{1,2}=\frac{-p \pm \sqrt{p^{2}-4\left(q-y_{k}\right)}}{2} \\
\stackrel{\downarrow}{\rightarrow} \& g(x)=x^{2}+p x+q-y=0 \stackrel{\text { Res. }}{\longrightarrow} \sum_{j=0}^{4} Y_{j} y^{j}=0 \stackrel{Y_{3}=0}{\longrightarrow} y^{4}+Y_{2} y^{2}+Y_{0}=0 \stackrel{(13)}{\longrightarrow} y_{k} \uparrow
\end{array}
$$

Figure 2. Flowchart of the steps to solve the Quartic Equation

Observe that undoing the $2^{\text {nd }}$ degree transformation $g(x)$ means that each value of the resultant $y$, gives rise to two values in $x$. See some details of this procedure:

The resultant of $f(x) \& g(x)$, is: $f(y)=y^{4}+Y_{3} y^{3}-Y_{2} y^{2}+Y_{1} y-Y_{0}=0$

$$
\text { where, }\left\{\begin{array}{c}
Y_{3}=c-2 q \\
Y_{2}=-\left(c p^{2}+c^{2}+3 d p-6 c q+6 q^{2}+2 e\right) \\
Y_{1}=d p^{3}-2 c p^{2} q+c d p+4 e p^{2}-2 c^{2} q-6 d p q+6 c q^{2}-4 q^{3}-d^{2}+2 c e-4 e q \\
Y_{0}=-\left(e p^{4}-d p^{3} q+c p^{2} q^{2}+c e p^{2}-c d p q-6 d p q-6 e p^{2} q+\cdots\right. \\
\left.\ldots+c^{2} q^{2}+3 d p q^{2}-2 c q^{3}+q^{4}-d e p+d^{2} q-2 c e q+2 e q^{2}+e^{2}\right)
\end{array}\right\}
$$

Making $Y_{3}=Y_{1}=0$ we obtain, the value $q=c / 2$ and three values of $p$ coming from a $3^{\text {rd }}$ degree equation. With $Y_{2}$ and $Y_{0}$ known we have: $y^{4}-Y_{2} y^{2}-Y_{0}=0$, whose roots, written directly from the general solution (13) for even DMF, are:

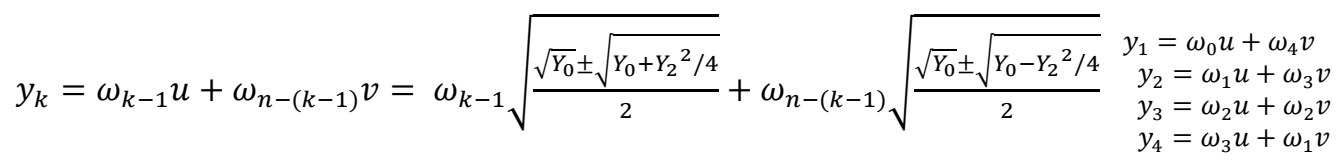

The roots $x_{i}$ of $\boldsymbol{g}(\boldsymbol{x})=\boldsymbol{x}^{2}+\boldsymbol{p} \boldsymbol{x}+\boldsymbol{q}-\boldsymbol{y}=\mathbf{0}$, become:

$$
\begin{aligned}
& x_{k}=\frac{-p \pm \sqrt{p^{2}-4\left(q-y_{k}\right)}}{2}=\frac{-p \pm \sqrt{p^{2}-4\left(q-\left(\omega_{k-1} u+\omega_{n-(k-1)} v\right)\right)}}{2}
\end{aligned}
$$

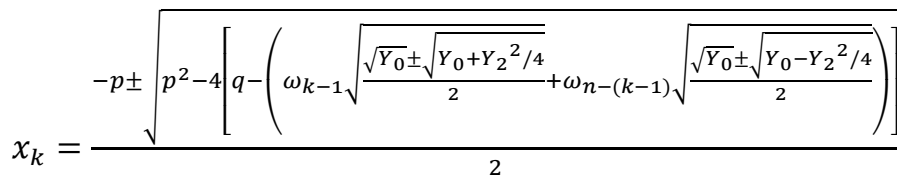

This would be a Cardano-style version of the solution of the quartic equation.

However, notice that the De Moivre quartic equation, $y^{4}-Y_{2} y^{2}-Y_{0}=0$, would also admit to be solved in a shorter way, making the change $y^{2}=z \rightarrow y= \pm \sqrt{z}$, reducing it 
to a quadratic equation, $z^{2}-Y_{2} z-Y_{0}=0$, which would greatly simplify its solution. See this formula not depending on $u$ and $v$ :

$$
y=\sqrt{Z}= \pm \sqrt{\frac{Y_{2} \pm \sqrt{Y_{2}{ }^{2}+4 Y_{0}}}{2}} \rightarrow x=\frac{-p \pm \sqrt{p^{2}-4(q-y)}}{2}=\frac{-p \pm \sqrt{p^{2}-4\left[q \mp \sqrt{\frac{Y_{2 \pm} \sqrt{Y_{2}{ }^{2}+4 Y_{0}}}{2}}\right]}}{2}
$$

In the first case, or Cardano's case, six sets of possibilities arise for the four roots of the quartic equation from the three values of $p$, and in the quadratic three sets arise, but only one is true: the one that returns the original equation, $x^{4}+c x^{2}+d x+e=0$; i.e., we have to check simultaneously all the possible solutions to see which one is valid.

\subsection{Solving the $5^{\text {th }}$ Degree Equation by radicals}

To do this, it is required to transform the Bring-Jerrard normal quintic equation (BJQ), $f(x)=x^{5}+q x+r=0$, to the De Moivre form in order to solve it.

To transform the BJQ to the De Moivre quintic form (DMQ), without the $2^{\text {nd }}$ and $4^{\text {th }}$ terms and with the relationship between the $3^{\text {rd }}$ and $5^{\text {th }}$ terms, $\left(Y_{3}\right)^{2}=5 Y_{l}$, we will use a new $4^{\text {th }}$ degree Tschirnhaus transformation, $g(x, y)=x^{4}+d x^{3}+c x^{2}+b x+a-y=0$, on the BJQ. For this purpose, we had to modify the expressions of the Bring coefficients as new ones: $\mathbf{a}=\mathbf{4 q} / \mathbf{5}, \mathbf{b}=\mathbf{f} \mathbf{d}+\mathbf{h}+\mathbf{g}$ and $\mathbf{c}=\mathbf{d}+\mathbf{h}+\mathbf{i}$. This added a new variable, necessary to eliminate $Y_{2}$ (instead of $Y_{3}$ ) and thus manage to construct the DMQ. After solving the De Moivre quintic equation obtained, $\mathrm{DMQ}$, in the process of undoing the previous transformation performed to the BJQ, we need the inverse formula that solves that equation, to have the expression of the original variable $x$. But for that, we will need a new $2^{\text {nd }}$ degree transformation $G(x, z)=x^{2}+m x+n-z=0$, on $g\left(x, y_{k}\right)$ to transform its resultant, $R_{2}\left(z\left(y_{k}\right)\right)=z^{4}+\sum_{1}^{4} Z_{4-j} z^{4-j}=0$, by making $Z_{3}=Z_{1}=0$ into a $4^{\text {th }}$ degree $D e$ Moivre form, $z^{4}+Z_{2} z^{2}+Z_{0}=0$. Notice that, the four roots $z_{i}$ of it, will come out using the five roots $y_{k}$ of the first resultant of the solved $5^{\text {th }}$ degree De Moivre equation. Thus, the inverse formula of $G(x, z), \quad x=G^{-1}\left(z_{i}\left(y_{k}\right)\right)=-\frac{m}{2} \pm \frac{\sqrt{m^{2}-4\left(n-z_{i}\left(y_{k}\right)\right)}}{2}$, gives us two roots for $x$. Then in sum, these two values of $x\left(z_{i}\left(y_{k}\right)\right)$, depend on the four values of $z\left(y_{k}\right)$. But, only one value of $x$ (depending on $y_{k}$ ) out of the possible ones, $4 \times 2=8$, shall satisfy the original BJQ, $f(x)=x^{5}+q x+r=0$. The values of $\boldsymbol{m}\left(y_{k}\right)$ and $\boldsymbol{n}\left(y_{k}\right)$ which satisfy the BJQ will be chosen. Although we will go deeper into this process, the changes made and undone for solving the BJQ are summarized as follows:

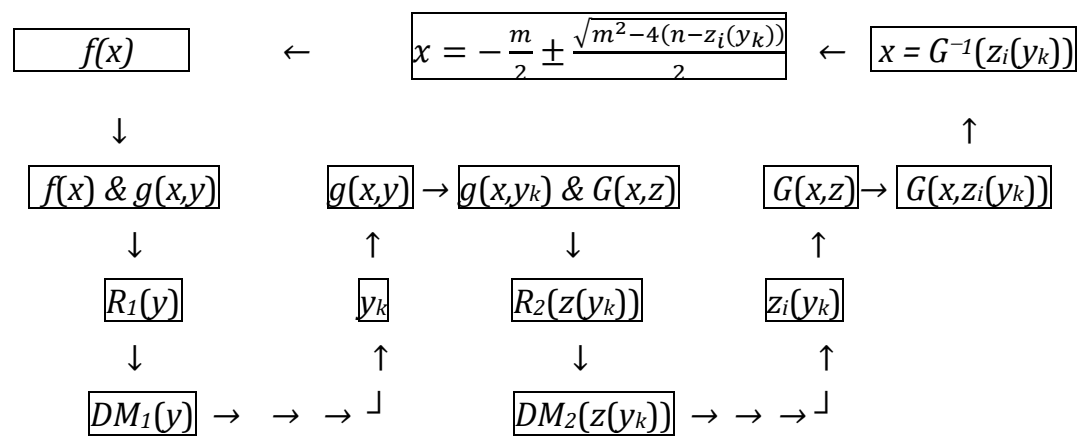

Figure 3. Flowchart of the steps to solve the Bring-Jerrard Normal Quintic Equation (BJQ)

Next are some details of this procedure by using the free open-source mathematics software system Sagemath (https://www.sagemath.org/). Let us start performing the $4^{\text {th }}$ degree transformation $(g(x, y))$ on the BJQ equation $(f(x))$ to obtain its resultant. 
$\mathrm{cb}=\mathrm{x}^{\wedge} 5+\mathrm{q}^{*} \mathrm{x}+\mathrm{r}$

res $=$ cb.resultant $\left(-y+x^{\wedge} 4+d^{*} x^{\wedge} 3+c^{*} x^{\wedge} 2+b^{*} x+a, x\right) \cdot \operatorname{poly}(y)$

As said, the modified Bring expressions of the coefficients $c, b$ and a, present in the 4th degree transformation applied to the BJQ, reduced the order of auxiliary equations to degrees less than 5, and, allowed us to obtain the desired expression of the DMQ:

$$
c=d+h+i, \quad b=f^{*} d+h+g, \quad a=4^{*} q / 5
$$

(Recall that coefficients used by E. S. Bring in his original $4^{\text {th }}$ degree transformation on the principal form to obtain the BJQ were: $\boldsymbol{c}=\boldsymbol{d}+\boldsymbol{\gamma}, \boldsymbol{b}=\boldsymbol{\sigma d}+\boldsymbol{\zeta}$ and $\boldsymbol{a}=(3 \boldsymbol{p d}+\mathbf{4 q ) / 5})$.

The resultant equation, for $Y_{4}=0$ by doing $a=4 q / 5$, can be written as: $R_{1}(y)=y^{5}+$ $\sum_{2}^{5} Y_{5-j} y^{5-j}=y^{5}+Y_{3} y^{3}+Y_{2} y^{2}+Y_{1} y+Y_{0}=0$, where:

$$
\begin{aligned}
& Y_{3}=1 / 5\left(5 d^{2}(2 q+5 r)+20 d g q+40 d h q+10 h^{2} q+20 d i q+20 h i q+10 i^{2} q\right. \\
& \left.+25 d h r+25 d i r+5\left(4 d^{2} q+5 d r\right) f-2 q^{2}+25 g r+25 h r\right) \\
& Y_{2}=-1 / 25\left(25 d^{3} f^{2}(4 q+5 r)+100 d^{2} f^{2} h q+100 d^{2} f^{2} i q+200 d^{2} f g q+200 d^{2} f h q\right. \\
& +200 d f g h q+200 d f h^{2} q+200 d f g i q+200 d f h i q-100 d^{2} h q^{2} \\
& -100 d^{2} i q^{2}+250 d^{2} f g r+500 d^{2} f h r+125 d f h^{2} r+250 h^{2} f i r \\
& +250 d f h i r+125 d f i^{2} r-25\left(4 q^{2}+3 q r\right) d^{3}+100 d g^{2} q+200 d g h q \\
& +100 g^{2} h q+100 d h^{2} q+200 g h^{2} q+100 h^{3} q+100 g^{2} i q+200 g h i q \\
& +100 h^{2} i q-40 d g q^{2}-80 d h q^{2}-20 h^{2} q^{2}-40 d i q^{2}-40 h i q^{2} \\
& -20 i^{2} q^{2}+125 d^{2} g r+125 d g^{2} r+125 d^{2} h r+500 d g h r+375 d h^{2} r \\
& +125 g h^{2} r+125 h^{3} r+250 d g i r+250 d h i r+250 \text { ghir }+250 h^{2} i r \\
& +125 g i^{2} r+125 h i^{2} r-250 d h q r-250 \text { diqr }-5\left(4 q^{2}+50 q r\right. \\
& \left.+25 r^{2}\right) d^{2}-4 q^{3}-25 g q r-25 h q r-125 d r^{2}-125 h r^{2}-125 i r^{2} \\
& \left.-5\left(8 d^{2} q^{2}-25 d^{3} r+5 d q r\right) f\right) \\
& Y_{1}=d^{4} f^{4} q+43 d^{4} f^{3} g q+4 d^{3} f^{3} h q+2 d^{4} f^{2} q^{2}+5 d^{4} f f^{3} r+5 d^{3} f^{3} h r+5 d^{3} f^{3} i r \\
& +6 d^{2} f^{2} g^{2} q+12 d^{2} f^{2} g h q+6 d^{2} f^{2} h^{2} q-4 d^{4} f q^{2}-12 / 5 d^{3} f^{2} q^{2} \\
& +4 d^{3} f g q^{2}-4 d^{3} f h q^{2}-12 / 5 d^{2} f^{2} h q^{2}-4 d^{2} f h^{2} q^{2}-8 d^{3} f i q^{2} \\
& -12 / 5 d^{2} f^{2} i q^{2}-8 d^{2} f h i q^{2}-4 d^{2} f i^{2} q^{2}+d^{4} q^{3}+15 d^{3} f^{2} g r \\
& +15 d^{3} f^{2} h r+15 d^{2} f^{2} g h r+15 d^{2} f^{2} h^{2} r+15 d^{2} f^{2} g i r+15 d^{2} f^{2} h i r \\
& -7 d^{4} f q r+5 d^{3} f^{2} q r-7 d^{3} f h q r-7 d^{3} f i q r-5 d^{4} f r^{2}+4 d f g^{3} q \\
& +12 d f g^{2} h q+12 d f g h^{2} q+4 d f h^{3} q+d^{4} q^{2}-4 d^{3} g q^{2} \\
& -24 / 5 d^{2} f g q^{2}+2 d^{2} g^{2} q^{2}-24 / 5 d^{2} f h q^{2} \\
& -4 d^{2} g h q^{2}-24 / 5 d f g h q^{2}-24 / 5 d f h^{2} q^{2}-4 d g h^{2} q^{2}+h^{4} q^{2}+ \\
& 4 d^{3} i q^{2}-8 d^{2} g i q^{2}-24 / 5 d f g i q^{2}+4 d^{2} h i q^{2}-24 / 5 d f h i q^{2}- \\
& 8 d g h i q^{2}+4 d h^{2} i q^{2}+4 h^{3} i q^{2}+6 d^{2} i^{2} q^{2}-4 d g i^{2} q^{2}+8 d h i^{2} q^{2}+ \\
& 6 h^{2} i^{2} q^{2}+4 d i^{3} q^{2}+4 h i^{3} q^{2}+i^{4} q^{2}+12 / 5 d^{3} q^{3}-28 / 25 d^{2} f q^{3}+ \\
& 12 / 5 d^{2} h q^{3}+12 / 5 d^{2} i q^{3}+15 d^{2} f g^{2} r+30 d^{2} f g h r+15 d f g^{2} h r+ \\
& 15 d^{2} f h^{2} r+30 d f g h^{2} r+15 d f h^{3} r+15 d f g^{2} i r+30 d f g h i r+ \\
& 15 d f h^{2} i r+d^{4} q r-5 d^{3} f q r-g q r+10 d^{2} f g q r-4 d^{3} h q r- \\
& 7 d^{2} g h q r-4 d^{2} h^{2} q r-5 d f h^{2} q r+d h^{3} q r+3 d^{3} i q r-10 d^{2} f i q r- \\
& 7 d^{2} \text { giqr }-d^{2} h i q r-10 d f h i q r+3 d h^{2} i q r+3 d^{2} i^{2} q r-5 d f i^{2} q r+ \\
& 3 d h h^{2} q r+d i^{3} q r+29 / 5 d^{3} q^{2} r+5 d^{4} r^{2}-5 d^{3} f r^{2}+5 d^{2} f^{2} r^{2}- \\
& 5 d^{3} g r^{2}+5 d^{3} h r^{2}-5 d^{2} f h r^{2}+5 d^{2} h^{2} r^{2}+10 d^{3} i r^{2}-5 d^{2} f i r^{2}+
\end{aligned}
$$




$$
\begin{aligned}
& 10 d^{2} h i r^{2}+5 d^{2} i^{2} r^{2}+g^{4} q+4 g^{3} h q+6 g^{2} h^{2} q+4 g h^{3} q+h^{4} q- \\
& 12 / 5 d g^{2} q^{2}-24 / 5 d g h q^{2}-12 / 5 g^{2} h q^{2}-12 / 5 d h^{2} q^{2}-24 / \\
& 5 g h^{2} q^{2}-12 / 5 h^{3} q^{2}-12 / 5 g^{2} i q^{2}-24 / 5 g h i q^{2}-12 / 5 h^{2} i q^{2}- \\
& 14 / 25 d^{2} q^{3}-28 / 25 d g q^{3}-56 / 25 d h q^{3}-14 / 25 h^{2} q^{3}-28 / \\
& 25 d i q^{3}-28 / 25 h i q^{3}-14 / 25 i^{3} q^{3}+5 d g^{3} r+15 d g^{2} h r+5 g^{3} h r+ \\
& 15 d g h^{2} r+15 g^{2} h^{2} r+5 d h^{3} r+15 g h^{3} r+5 h^{4} r+5 g^{3} i r+ \\
& 15 g^{2} h i r+15 g h^{2} i r+5 h^{3} i r-5 d^{2} g q r+5 d g^{2} q r-5 d^{2} h q r- \\
& 5 d h^{2} q r-5 g h^{2} q r-5 h^{3} q r-10 d g i q r-10 d h i q r-10 g h i q r- \\
& 10 h^{2} i q r-5 g i^{2} q r-5 h i^{2} q r+17 / 5 d^{2} q^{2} r-d f q^{2} r+17 / 5 d h q^{2} r+ \\
& 17 / 5 d i q^{2} r-5 d^{3} r^{2}-5 d^{2} g r^{2}+10 d f g r^{2}-20 d^{2} h r^{2}+10 d f h r^{2}- \\
& 5 d g h r^{2}-20 d h^{2} r^{2}-5 h^{3} r^{2}-15 d^{2} i r^{2}-5 d g i r^{2}-35 d h i r^{2}- \\
& 15 h^{2} i r^{2}-15 d i^{2} r^{2}-15 h i^{2} r^{2}-5 i^{3} r^{2}+9 d^{2} q r^{2}-3 / 125 q^{4}- \\
& g q^{2} r-h q^{2} r+5 g^{2} r^{2}+10 g h r^{2}+5 h^{2} r^{2}+2 d q r^{2}+2 h q r^{2}+ \\
& 2 i q r^{2}+5 d r^{3}
\end{aligned}
$$

The other factor $Y_{0}$ will not be needed for now. As next step we will expand the coefficient $Y_{2}$ in factors of the variable $d$, to obtain:

$$
\begin{gathered}
Y_{2}=D_{3} d^{3}+D_{2} d^{2}+D_{1} d+D_{0}=0 \rightarrow\left(D_{3}=D_{2}=D_{1}=D_{0}=0\right) \\
D_{3}=-\left(4 f^{2} q+5 f^{2} r-4 q^{2}+5 f r-3 q r\right) \\
D_{2}=-1 / 5\left(20 f^{2} h q+20 f^{2} i q+40 f g q+40 f h q-8 f q^{2}-20 h q^{2}-20 i q^{2}+50 f g r\right. \\
\left.+100 f h r+50 f i r-4 q^{2}+25 g r+25 h r-50 q r-25 r^{2}\right) \\
D_{1}=-1 / 5\left(40 f g h q+40 f h^{2} q+40 f g i q+40 f h i q+25 f h^{2} r+50 f h i r+25 f i^{2} r\right. \\
+20 g^{2} q+40 g h q+20 h^{2} q-8 g q^{2}-16 h q^{2}-8 i q^{2}+25 g^{2} r \\
+100 g h r+75 h^{2} r+50 g i r+50 h i r-5 f q r-50 h q r-50 i q r \\
\left.-25 r^{2}\right) \\
-8 f h^{2} q-8 f g i q-8 f h i q-5 f h^{2} r-10 f h i r-5 f i^{2} r-4 g^{2} \\
-8 g h q-4 h^{2} q+8 / 5 g q^{2}+16 / 5 h q^{2}+8 / 5 i q^{2}-5 g^{2} r-20 g h r \\
-15 h^{2} r-10 g i r-10 h i r+f q r+10 h q r+10 i q r+5 r^{2}
\end{gathered}
$$

The Sagemath command, to solve equations used to obtain the values of $f, g, h$ and $i$, is:

solve $(\mathrm{D} 3==0, \mathrm{f})$

$f=-1 / 2\left(5 r \pm \operatorname{sqrt}\left(64 q^{3}+128 q^{2} r+5(12 q+5) r^{2}\right)\right) /(4 q+5 r)$,

Notice that any of the two solutions of the variable $f$ is a number, since $f$ depends only on the known coefficients $q$ and $r$. From now on, we will refer to $f$ as another known constant number, in addition to $q$ and $r$.

$$
\begin{aligned}
& \text { solve(D2==0,g) } \\
& \qquad \begin{array}{c}
g=1 / 5\left(4(2 f+5 \boldsymbol{h}+5 \boldsymbol{i}+1) q^{2}-20\left(f^{2} \boldsymbol{i}+\left(f^{2}+2 f\right) \boldsymbol{h}\right) q-25((4 f+1) \boldsymbol{h}+2 f \boldsymbol{i}\right. \\
\left.-2 q) r+25 r^{2}\right) /(8 f q+5(2 f+1) r)
\end{array}
\end{aligned}
$$

Note that in this result, the variable $g$ becomes linearly dependent (first-degree) on the unknown variables $h$ and $i$. A simplified expression of $g$ could be then: $g=P \boldsymbol{h}+$ $Q \boldsymbol{i}+R$ where $P, Q$ and $R$ become numerical factors. Substituting the not simplified $g$, namely, the original $g$, into the expression of $D_{1}$, we obtain a function dependent on $h$ and $i$. Let's call it D1H. Putting D1H as an equation in $h$, equal to zero, results in a $2^{\text {nd }}$ degree equation. Solving it we obtain $h$ : 


$$
\begin{aligned}
& \text { solve }(\mathrm{D} 1 \mathrm{H}==0, \mathrm{~h}) \\
& h=-1 / 5\left(960 f^{4} \boldsymbol{i} q^{3}-64(5 \boldsymbol{i}+1) q^{5}-64\left(4 f^{3}+10 f^{2} \boldsymbol{i}-3 f^{2}\right) q^{4}-125\left(5 \left(4 f^{3}\right.\right.\right. \\
& \left.-3 f) \boldsymbol{i}-\left(16 f^{2}+16 f-5\right) q+4 q^{2}\right) r^{3}-625 r^{4}+200\left(15 f^{2} \boldsymbol{i} q\right. \\
& \left.+\left(13 f^{2}+5 f-5 i\right) q^{2}-7 q^{3}\right) r^{2}-80\left(5(\boldsymbol{i}+2) q^{4}-15\left(f^{4}\right.\right. \\
& \left.\left.+2 f^{3}\right) \boldsymbol{i} q^{2}+\left(4 f^{3}-10 f^{2}+10\left(f^{2}+f\right) \boldsymbol{i}-3 f\right) q^{3}\right) r \pm \boldsymbol{s q r t}\left(\mathbf { 5 1 2 } \left(\boldsymbol{f}^{2}\right.\right. \\
& +f-1) q^{7}+256 q^{8}+256\left(f^{4}-6 f^{3}+3 f^{2}\right) q^{6}-15625(f \\
& -1) r^{6}+3125\left(20 f^{3}-2(8 f-5) q-15 f\right) r^{5}+625\left(\left(76 f^{2}+40 f\right.\right. \\
& \left.+65) q^{2}-40 q^{3}+5\left(4 f^{4}-27 f^{2}\right) q\right) r^{4}+15000\left(\left(6 f^{2}+5 f\right) q^{3}\right. \\
& \left.-4 q^{4}-\left(2 f^{4}+5 f^{3}\right) q^{2}\right) r^{3}+400\left((13 f-88) q^{5}-3\left(6 f^{3}-50 f^{2}\right.\right. \\
& \left.-9 f) q^{4}-15\left(f^{5}+6 f^{4}\right) q^{3}\right) r^{2}-320\left(15 f^{5} q^{4}-(25 f-28)\right. \\
& \left.\left.\left.+q^{6}+\left(14 f^{3}-24 f^{2}-3 f\right) q^{5}\right) r\right)(8 f q+5(2 f+1) r)\right) /\left(192 f^{4} q^{3}\right. \\
& -128 f^{2} q^{4}-64 q^{5}-125\left(4 f^{3}-3 f\right) r^{3}+200\left(3 f^{2} q-q^{2}\right) r^{2} \\
& \left.-80\left(2\left(f^{2}+f\right) q^{3}+q^{4}-3\left(f^{4}+2 f^{3}\right) q^{2}\right) r\right),
\end{aligned}
$$

Notice that obtained roots, $h_{1}$ and $h_{2}$, both depend linearly (first-degree) on $i$, since the expression inside the square root present in each $h_{i}$ depends only on the known numbers $f, q$ and $r$. This quadratic result of $h$ could be then simplified to a linear expression something like: $h=S+T^{*} i \pm U$, with numerical values for $S, T$ and $U$.

The character of this 2 nd degree equation, of giving roots linearly dependent on the variable $i$ causes that, instead of being 6 the final degree, $2 \times 3=6$, becomes $1 \times 3=3<5$. This crucial and unexpected feature determines the resolution of the quintic, punching a hole in the Ruffini, Abel and Galois theorems on the impossibility of solving it. Let us check it:

Substituting the chosen value of $h$ in the unknown $g$; and then $h$ and $g$ in $D_{0}$, we obtain a function D0I (dependent only on $i$ ). Making DOI equal to zero, we obtain a 3rd degree solvable equation. We solve it and have its three roots in numerical form. We shall choose that $i$ that returns the original Bring equation.

Substituting now the numerical value of $i$, in $g$ and in $h$, we would have completed the numerical values of $f, g, h$ and $i$, thus eliminating $Y_{2}$. Next, these numerical values of $f, g, h$ and $i$ are entered into the expressions of $a, b$ and $c$ in $Y_{3}, Y_{1}$ and $Y_{0}$.

At this time, it only remains to ensure that the relation $\left(Y_{3}\right)^{2}=5 Y_{1}$ is satisfied, which depends only on the variable $d$ (the only remaining unknown). Expressing it as an equation, $\left(Y_{3}\right)^{2}-5 Y_{1}=0$, we obtain a $4^{\text {th }}$ degree equation in $d$. We solve it and choose that root out of the four, $d_{1}, d_{2}, d_{3}$, $d_{4}$, satisfying the relation $\left(Y_{3}\right)^{2}=5 Y_{1}$, that gives us back the original Bring equation, $x^{5}+q x+r$. Thus, with $Y_{4}=Y_{2}=0$, the resultant $R_{1}(y)$ is transformed into another equation with the De Moivre structure, $y^{5}+Y_{3} y^{3}+Y_{1} y+Y_{0}=0$, with all its coefficients already known, meeting the relation $\left(Y_{3}\right)^{2}=5 Y_{1}$, and solvable through the general formula shown in equation (10), for quintics. Let's write it:

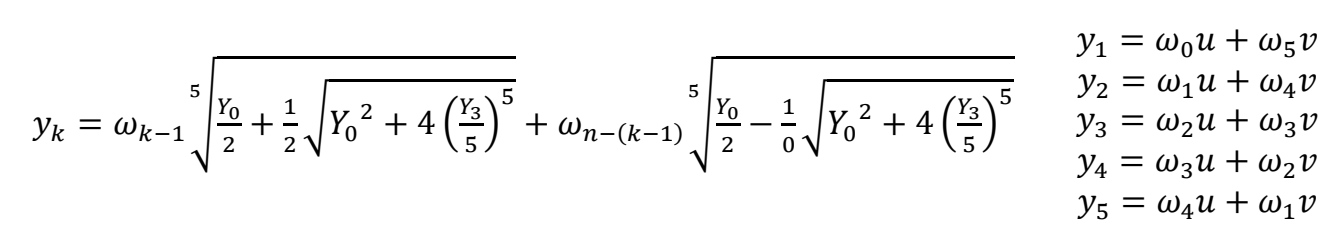

Thus, we have succeeded in transforming any BJQ (and hence to any general quintic equation) to the radically soluble De Moivre form, fulfilling our main goal of demonstrating that any quintic is radically soluble.

Next, we need to undo, first, the $4^{\text {th }}$ degree transformation, $g(x)=x^{4}+d x^{3}+$ $c x^{2}+b x+a-y=0$, applied to the Bring equation $x^{5}+q x+r=0$, up to the original roots $x\left(y_{k}\right)$ of the quintic, depending on the five roots $y_{k}=\omega_{k-1} u+\omega_{(n-k-1)} v$. Although simple, it is a long process and involves obtaining the inverse formula of 
$g(x)$. To do so, $g(x)$ must be transformed also into a De Moivre quartic by a $2^{\text {nd }}$ degree transformation, $G(x, z)=x^{2}+m x+n-z=0$. Let's see:

$$
\begin{aligned}
& g\left(x, y_{k}\right)=x^{4}+d x^{3}+c x^{2}+b x+a-y_{k}=0 \& G(x, z)=x^{2}+m x+n-z=0 \quad \rightarrow \ldots \\
& \therefore \rightarrow R_{2}\left(z\left(y_{k}\right)\right)=z^{4}+\sum_{1}^{4} Z_{4-j} z^{4-j}=0 \stackrel{Z_{3}=z_{1}=0}{\longrightarrow} D M_{2}\left(z\left(y_{k}\right)\right)=z^{4}+Z_{2} z^{2}+Z_{0}=0 .
\end{aligned}
$$

Figure 4. Flowchart of the steps to solve the quartic transformation for solving the Quintic

Where, $m, n, Z_{2}$ and $Z_{0}$ (by making $Z_{3}=0=Z_{1}$ ) become known as function of the roots $y_{k}$. Moreover, by making a new change $w=z^{2} \rightarrow z=\sqrt{w}$, a simpler equation, $w^{2}+Z_{2} w+Z_{0}=0$, is obtained. Thus, four roots for $z\left(y_{k}\right)$ arise $(2 \times 2=4)$. This means that undoing up to $x$, using all possible values of variables $f_{12}, g_{1}, h_{12}, i_{123}$, $d_{1234}, m_{123}, n_{1}, w_{12}, z_{12}$, and $x_{12}$ requires many simultaneous calculations, only one satisfying the original Bring equation, $x^{5}+q x+r=0$.

$$
\begin{aligned}
& \operatorname{var}(' a, b, c, d, e, f, g, h, i, j, k, 1, m, n, x, y, z, p, q, r, s, t, u, v, T, K, L, M, N ') \\
& c b=x^{\wedge} 4+d^{*} x^{\wedge} 3+c^{*} x^{\wedge} 2+b^{*} x+a-y \\
& \operatorname{res}=\operatorname{cb} \cdot \operatorname{resultant}\left(x^{\wedge} 2+m^{*} x+\left(-d^{\wedge} 2+d^{*} m+2^{*} c\right) / 4-z, x\right) \cdot \operatorname{poly}(z)
\end{aligned}
$$

The resultant with $Z_{3}=0$, for $n=-d^{2}+d m+2 c / 4$, is: $z^{4}+Z_{2} z^{2}+Z_{1} z+Z_{0}=0$ :

$$
\begin{aligned}
Z_{2}=1 / 8\left(3 d^{4}-\right. & 6 d^{3} m+3 d^{2} m^{2}-12 c d^{2}+20 c d m-8 c m^{2}+4 c^{2}+ \\
& 16 b d-24 b m-16 a+16 y) \\
Z_{1}=-1 / 8\left(d^{6}-\right. & 6 c d^{4}+8 b d^{3}-\left(d^{3}-4 c d+8 b\right) m^{3}-16 b c d+8\left(c^{2}+y\right) d^{2} \\
& -8 a d^{2}+\left(3 d^{4}-14 c d^{2}+8 c^{2}+20 b d-32 a+32 y\right) m^{2}+8 b^{2} \\
& \left.-\left(3 d^{5}-16 c d^{3}+20 b d^{2}-16 b c+16\left(c^{2}+2 y\right) d-32 a d\right) m\right) \\
Z_{0}=-3 / 256 d^{8}+ & 3 / 64 d^{7} m-9 / 128 d^{6} m^{\wedge} 2+3 / 64 d^{5} m^{\wedge} 3+3 / 32 c d^{6} \\
& -11 / 32 c d^{5} m+15 / 32 c d^{4} m^{2}-9 / 32 c d^{3} m^{3}+11 / 16 c^{2} d^{3} m \\
+ & 7 / 16 b d^{4} m-23 / 32 c^{2} d^{2} m^{2}-3 / 4 b d^{3} m^{2}+1 / 4 c^{2} d m^{3} \\
+ & 11 / 16 b d^{2} m^{3}+1 / 8 c^{3} d^{2}-3 / 8 c^{3} d m-b c d^{2} m-a d^{3} m \\
+ & 1 / 4 c^{3} m^{2}+b c d m^{2}+15 / 8 a d^{2} m^{2}-1 / 2 b c m^{3}-2 a d m^{3} \\
- & 1 / 256\left(3 d^{4}-16 c d^{2}+64 b d-256 a+256 y\right) m^{4}-1 / 8 d^{4} y \\
+ & d^{3} m y-15 / 8 d^{2} m^{2} y+2 d m^{3} y+1 / 16 c^{4}+1 / 4 b c^{2} m+1 / 4 b^{2} d m \\
+ & 3 / 2 a c d m-a c m^{2}-3 / 2 c d m y+c m^{2} y-1 / 4\left(d^{2}-2 c\right) b^{2} \\
& -1 / 32\left(7 d^{4}+16 a-16 y\right) c^{2}-a b m+b m y+1 / 8\left(d^{4}-16 y\right) a+a^{2} \\
& -1 / 8\left(d^{5}-4 c d^{3}+4 c^{2} d\right) b+y^{2}
\end{aligned}
$$

Making $Z_{1}=0$, we obtain a $3^{\text {rd }}$ degree equation in $m$. This value depends on $y_{k}$.

$$
\begin{aligned}
m=-1 / 3(1 / 2)^{(2 / 3)} & (- \text { Isqrt }(3) \\
& +1)\left(\left(3 d^{4}-14 c d^{2}+8 c^{2}+20 b d-32 a+32 y\right)^{2} /\left(d^{3}-4 c d+8 b\right)^{2}\right. \\
& -3\left(3 d^{5}-16 c d^{3}+16 c^{2} d+4\left(5 d^{2}-4 c\right) b-32 a d+32 d y\right) /\left(d^{3}-4 c d\right. \\
& +8 b)) /
\end{aligned}
$$




$$
\begin{aligned}
& \left(2\left(3 d^{4}-14 c d^{2}+8 c^{2}+20 b d-32 a+32 y\right)^{3} /\left(d^{3}-4 c d+8 b\right)^{3}\right. \\
& -9\left(3 d^{5}-16 c d^{3}+16 c^{2} d+4\left(5 d^{2}-4 c\right) b-32 a d+32 d y\right)\left(3 d^{4}\right. \\
& \left.-14 c d^{2}+8 c^{2}+20 b d-32 a+32 y\right) /\left(d^{3}-4 c d+8 b\right)^{2}+27\left(d^{6}-6 c d^{4}\right. \\
& \left.+8 c^{2} d^{2}-8 a d^{2}+8 d^{2} y+8\left(d^{3}-2 c d\right) b+8 b^{2}\right) /\left(d^{3}-4 c d+8 b\right) \\
& +72 \operatorname{sqrt}\left(9 a^{2} d^{4}+4 / 3 b^{2} c^{3}-16 / 3 a c^{4}+9 b^{4}-48 a b^{2} c+128 / 3 a^{2} c^{2}\right. \\
& +2 / 3\left(2 b^{3}-9 a b c\right) d^{3}-256 / 3 a^{3}-1 / 3\left(b^{2} c^{2}-4 a c^{3}-6 a b^{2}+144 a^{2} c\right) d^{2} \\
& +1 / 3\left(27 d^{4}-144 c d^{2}+128 c^{2}+192 b d-768 a\right) y^{2}+256 / 3 y^{3} \\
& -2 / 3\left(9 b^{3} c-40 a b c^{2}-96 a^{2} b\right) d+2 / 3\left(9 b c d^{3}-27 a d^{4}+8 c^{4}+72 b^{2} c\right. \\
& \left.\left.-128 a c^{2}-\left(2 c^{3}+3 b^{2}-144 a c\right) d^{2}+384 a^{2}-8\left(5 b c^{2}+24 a b\right) d\right) y\right) /\left(d^{3}\right. \\
& -4 c d+8 b))^{(1 / 3)}-1 / 6(1 / 2)^{(1 / 3)}(\operatorname{Isqrt}(3)+1) \\
& +\left(2\left(3 d^{4}-14 c d^{2}+8 c^{2}+20 b d-32 a+32 y\right)^{3} /\left(d^{3}-4 c d+8 b\right)^{3}\right. \\
& -9\left(3 d^{5}-16 c d^{3}+16 c^{2} d+4\left(5 d^{2}-4 c\right) b-32 a d+32 d y\right)\left(3 d^{4}\right. \\
& \left.-14 c d^{2}+8 c^{2}+20 b d-32 a+32 y\right) /\left(d^{3}-4 c d+8 b\right)^{2}+27\left(d^{6}\right. \\
& \left.-6 c d^{4}+8 c^{2} d^{2}-8 a d^{2}+8 d^{2} y+8\left(d^{3}-2 c d\right) b+8 b^{2}\right) /\left(d^{3}-4 c d\right. \\
& +8 b)+72 \operatorname{sqrt}\left(9 a^{2} d^{4}+4 / 3 b^{2} c^{3}-16 / 3 a c^{4}+9 b^{4}-48 a b^{2} c\right. \\
& +128 / 3 a^{2} c^{2}+2 / 3\left(2 b^{3}-9 a b c\right) d^{3}-256 / 3 a^{3}-1 / 3\left(b^{2} c^{2}-4 a c^{3}\right. \\
& \left.-6 a b^{2}+144 a^{2} c\right) d^{2}+1 / 3\left(27 d^{4} 4-144 c d^{2}+128 c^{2}+192 b d\right. \\
& -768 a) y^{2}+256 / 3 y^{3}-2 / 3\left(9 b^{3} c-40 a b c^{2}-96 a^{2} b\right) d+2 / 3\left(9 b c d^{3}\right. \\
& -27 a d^{4}+8 c^{4}+72 b^{2} c-128 a c^{2}-\left(2 c^{3}+3 b^{2}-144 a c\right) d^{2}+384 a^{2} \\
& \left.\left.\left.-8\left(5 b c^{2}+24 a b\right) d\right) y\right) /\left(d^{3}-4 c d+8 b\right)\right)^{(1 / 3)}+1 / 3\left(3 d^{4}-14 c d^{2}\right. \\
& \left.+8 c^{2}+20 b d-32 a+32 y\right) /\left(d^{3}-4 c d+8 b\right), \\
& m=-1 / 3(1 / 2)^{\left(\frac{2}{3}\right)}(\operatorname{Isqrt}(3) \\
& +1)\left(\left(3 d^{4}-14 c d^{2}+8 c^{2}+20 b d-32 a+32 y\right)^{2} /\left(d^{3}-4 c d+8 b\right)^{2}\right. \\
& -3\left(3 d^{5}-16 c d^{3}+16 c^{2} d+4\left(5 d^{2}-4 c\right) b-32 a d+32 d y\right) /\left(d^{3}-4 c d\right. \\
& +8 b)) \\
& /\left(2\left(3 d^{4}-14 c d^{2}+8 c^{2}+20 b d-32 a+32 y\right)^{3} /\left(d^{3}-4 c d+8 b\right)^{3}-9\left(3 d^{5}\right.\right. \\
& \left.-16 c d^{3}+16 c^{2} d+4\left(5 d^{2}-4 c\right) b-32 a d+32 d y\right)\left(3 d^{4}-14 c d^{2}+8 c^{2}\right. \\
& +20 b d-32 a+32 y) /\left(d^{3}-4 c d+8 b\right)^{2}+27\left(d^{6}-6 c d^{4}+8 c^{2} d^{2}\right. \\
& \left.-8 a d^{2}+8 d^{2} y+8\left(d^{3}-2 c d\right) b+8 b^{2}\right) /\left(d^{3}-4 c d+8 b\right) \\
& +72 \operatorname{sqrt}\left(9 a^{2} d^{4}+4 / 3 b^{2} c^{3}-16 / 3 a c^{4}+9 b^{4}-48 a b^{2} c+128 / 3 a^{2} c^{2}\right. \\
& +2 / 3\left(2 b^{3}-9 a b c\right) d^{3}-256 / 3 a^{3}-1 / 3\left(b^{2} c^{2}-4 a c^{3}-6 a b^{2}+144 a^{2} c\right) d^{2} \\
& +1 / 3\left(27 d^{4}-144 c d^{2}+128 c^{2}+192 b d-768 a\right) y^{2}+256 / 3 y^{3} \\
& -2 / 3\left(9 b^{3} c-40 a b c^{2}-96 a^{2} b\right) d+2 / 3\left(9 b c d^{3}-27 a d^{4}+8 c^{4}+72 b^{2} c\right. \\
& \left.\left.-128 a c^{2}-\left(2 c^{3}+3 b^{2}-144 a c\right) d^{2}+384 a^{2}-8\left(5 b c^{2}+24 a b\right) d\right) y\right) /\left(d^{3}\right. \\
& -4 c d+8 b))^{\left(\frac{1}{3}\right)}
\end{aligned}
$$




$$
\begin{aligned}
& -1 / 6(1 / 2)^{\left(\frac{1}{3}\right)}(\operatorname{Isqrt}(3) \\
& +1)\left(2\left(3 d^{4}-14 c d^{2}+8 c^{2}+20 b d-32 a+32 y\right)^{3} /\left(d^{3}-4 c d+8 b\right)^{3}\right. \\
& -9\left(3 d^{5}-16 c d^{3}+16 c^{2} d+4\left(5 d^{2}-4 c\right) b-32 a d+32 d y\right)\left(3 d^{4}\right. \\
& \left.-14 c d^{2}+8 c^{2}+20 b d-32 a+32 y\right) /\left(d^{3}-4 c d+8 b\right)^{2}+27\left(d^{6}\right. \\
& \left.-6 c d^{4}+8 c^{2} d^{2}-8 a d^{2}+8 d^{2} y+8\left(d^{3}-2 c d\right) b+8 b^{2}\right) /\left(d^{3}-4 c d\right. \\
& +8 b)+72 \operatorname{sqrt}\left(9 a^{2} d^{4}+4 / 3 b^{2} c^{3}-16 / 3 a c^{4}+9 b^{4}-48 a b^{2} c\right. \\
& +128 / 3 a^{2} c^{2}+2 / 3\left(2 b^{3}-9 a b c\right) d^{3}-256 / 3 a^{3}-1 / 3\left(b^{2} c^{2}-4 a c^{3}\right. \\
& \left.-6 a b^{2}+144 a^{2} c\right) d^{2}+1 / 3\left(27 d^{4} 4-144 c d^{2}+128 c^{2}+192 b d\right. \\
& -768 a) y^{2}+256 / 3 y^{3}-2 / 3\left(9 b^{3} c-40 a b c^{2}-96 a^{2} b\right) d+2 / 3\left(9 b c d^{3}\right. \\
& -27 a d^{4}+8 c^{4}+72 b^{2} c-128 a c^{2}-\left(2 c^{3}+3 b^{2}-144 a c\right) d^{2}+384 a^{2} \\
& \left.\left.\left.-8\left(5 b c^{2}+24 a b\right) d\right) y\right) /\left(d^{3}-4 c d+8 b\right)\right)^{(1 / 3)}+1 / 3\left(3 d^{4}-14 c d^{2}\right. \\
& \left.+8 c^{2}+20 b d-32 a+32 y\right) /\left(d^{3}-4 c d+8 b\right) \text {, } \\
& m=2 / 3(1 / 2)^{\left(\frac{2}{3}\right)}\left(\left(3 d^{4}-14 c d^{2}+8 c^{2}+20 b d-32 a+32 \boldsymbol{y}\right)^{2} /\left(d^{3}-4 c d+8 b\right)^{2}\right. \\
& -3\left(3 d^{5}-16 c d^{3}+16 c^{2} d+4\left(5 d^{2}-4 c\right) b-+32 a d+32 d y\right) /\left(d^{3}\right. \\
& -4 c d+8 b)) / \\
& \left(2\left(3 d^{4}-14 c d^{2}+8 c^{2}+20 b d-32 a+32 y\right)^{3} /\left(d^{3}-4 c d+8 b\right)^{3}\right. \\
& -9\left(3 d^{5}-16 c d^{3}+16 c^{2} d+4\left(5 d^{2}-4 c\right) b-32 a d+32 d y\right)\left(3 d^{4}\right. \\
& \left.-14 c d^{2}+8 c^{2}+20 b d-32 a+32 y\right) /\left(d^{3}-4 c d+8 b\right)^{2}+27\left(d^{6}\right. \\
& \left.-6 c d^{4}+8 c^{2} d^{2} c-8 a d^{2}+8 d^{2} \boldsymbol{y}+8\left(d^{3}-2 c d\right) b+8 b^{2}\right) /\left(d^{3}-4 c d\right. \\
& +8 b)+72 \operatorname{sqrt}\left(9 a^{2} d^{4}+4 / 3 b^{2} c^{3}-16 / 3 a c^{4}+9 b^{4}-48 a b^{2} c\right. \\
& +128 / 3 a^{2} c^{2}+2 / 3\left(2 b^{3}-9 a b c\right) d^{3}-256 / 3 a^{3}-1 / 3\left(b^{2} c^{2}-4 a c^{3}-6 a b^{2}\right. \\
& \left.+144 a^{2} c\right) d^{2}+1 / 3\left(27 d^{4}-144 c d^{2}+128 c^{2}+192 b d-768 a\right) \boldsymbol{y}^{2} \\
& +256 / 3 \boldsymbol{y}^{3}-2 / 3\left(9 b^{3} c-40 a b c^{2}-96 a^{2} b\right) d+2 / 3\left(9 b c d^{3}-27 a d^{4}+8 c^{4}\right. \\
& +72 b^{2} c-128 a c^{2}-\left(2 c^{3}+3 b^{2}-144 a c\right) d^{2}+384 a^{2}-8\left(5 b c^{2}\right. \\
& \left.+24 a b) d) \boldsymbol{y}) /\left(d^{3}-4 c d+8 b\right)\right)^{(1 / 3)}+1 / 3(1 / 2)^{(1 / 3)} \\
& \left(2\left(3 d^{4}-14 c d^{2}+8 c^{2}+20 b d-32 a+32 y\right)^{3} /\left(d^{3}-4 c d+8 b\right)^{3}\right. \\
& -9\left(3 d^{5}-16 c d^{3}+16 c^{2} d+4\left(5 d^{2}-4 c\right) b-32 a d+32 d y\right)\left(3 d^{4}\right. \\
& \left.-14 c d^{2}+8 c^{2}+20 b d-32 a+32 y\right) /\left(d^{3}-4 c d+8 b\right)^{2}+27\left(d^{6}\right. \\
& \left.-6 c d^{4}+8 c^{2} d^{2}-8 a d^{2}+8 d^{2} \boldsymbol{y}+8\left(d^{3}-2 c d\right) b+8 b^{2}\right) /\left(d^{3}-4 c d\right. \\
& +8 b)+72 \operatorname{sqrt}\left(9 a^{2} d^{4}+4 / 3 b^{2} c^{3}-16 / 3 a c^{4}+9 b^{4}-48 a b^{2} c\right. \\
& +128 / 3 a^{2} c^{2}+2 / 3\left(2 b^{3}-9 a b c\right) d^{3}-256 / 3 a^{3}-1 / 3\left(b^{2} c^{2}-4 a c^{3}\right. \\
& \left.-6 a b^{2}+144 a^{2} c\right) d^{2}+1 / 3\left(27 d^{4} 4-144 c d^{2}+128 c^{2}+192 b d\right. \\
& -768 a) \boldsymbol{y}^{2}+256 / 3 \boldsymbol{y}^{3}-2 / 3\left(9 b^{3} c-40 a b c^{2}-96 a^{2} b\right) d+2 / 3\left(9 b c d^{3}\right. \\
& -27 a d^{4}+8 c^{4}+72 b^{2} c-128 a c^{2}-\left(2 c^{3}+3 b^{2}-144 a c\right) d^{2}+384 a^{2} \\
& \left.\left.\left.-8\left(5 b c^{2}+24 a b\right) d\right) y\right) /\left(d^{3}-4 c d+8 b\right)\right)^{(1 / 3)}+1 / 3\left(3 d^{4}-14 c d^{2}\right. \\
& \left.+8 c^{2}+20 b d-32 a+32 \boldsymbol{y}\right) /\left(d^{3}-4 c d+8 b\right)
\end{aligned}
$$

In this way, after solving $z^{4}+Z_{2} z^{2}+Z_{0}=0$, with their roots as function of $m\left(y_{k}\right)$ and $n\left(m\left(y_{k}\right)\right)$, namely $z=z\left(y_{k}\right)$, the undo process can be initiated. Starting from this equation and undoing its Tschirnhaus transformation to the original variable $x$, we have:

$$
Z^{4}+Z_{2} Z^{2}+Z_{0}=0 \rightarrow z= \pm \sqrt{\frac{-Z_{2} \pm \sqrt{\left(Z_{2}\right)^{2}-4\left(Z_{0}\right)}}{2}} \quad \rightarrow \quad x=\frac{-\boldsymbol{m} \pm \sqrt{\boldsymbol{m}^{2}-4(\boldsymbol{n}-\boldsymbol{z})}}{2}
$$

where, $Z_{2}$ and $Z_{0}$ depend on $m(y)$ and $y$ (for $y=y_{k}$ ): 


$$
\begin{aligned}
Z_{2}=-1 / 8\left(3 d^{4}\right. & -12 c d^{2}+\left(3 d^{2}-8 c\right) m^{2}+4 c^{2}+16 b d-2\left(3 d^{3}-10 c d\right. \\
& +12 b) m-16 a+16 y) \\
Z_{0}=-3 / 256 d^{8} & +3 / 32 c d^{6}-7 / 32 c^{2} d^{4}+1 / 8 c^{3} d^{2}-1 / 256\left(3 d^{4}-16 c d^{2}+64 b d\right. \\
& -256 a+256 y) m^{4}+1 / 16 c^{4}+1 / 64\left(3 d^{5}-18 c d^{3}+16 c^{2} d\right. \\
+ & \left.4\left(11 d^{2}-8 c\right) b-128 a d+128 d y\right) m^{3}-1 / 4\left(d^{2}-2 c\right) b^{2} \\
& -1 / 128\left(9 d^{6}-60 c d^{4}+92 c^{2} d^{2}-32 c^{3}\right. \\
& \left.-16\left(15 d^{2}-8 c\right) a+32\left(3 d^{3}-4 c d\right) b+16\left(15 d^{2}-8 c\right) \boldsymbol{y}\right) m^{2} \\
& +1 / 8\left(d^{4}-4 c^{2}-16 \boldsymbol{y}\right) a+a^{2}-1 / 8\left(d^{5}-4 c d^{3}+4 c^{2} d\right) b \\
& +1 / 64\left(3 d^{7}-22 c d^{5}+44 c^{2} d^{3}-24 c^{3} d+16 b^{2} d-32\left(2 d^{3}-3 c d\right.\right. \\
+ & \left.2 b) a+4\left(7 d^{4}-16 c d^{2}+4 c^{2}\right) b+32\left(2 d^{3}-3 c d+2 b\right) \boldsymbol{y}\right) m \\
& -1 / 8\left(d^{4}-4 c^{2}\right) \boldsymbol{y}+\boldsymbol{y}^{2}
\end{aligned}
$$

Then, these last results allow us to reach the inverse formula for the BJQ:

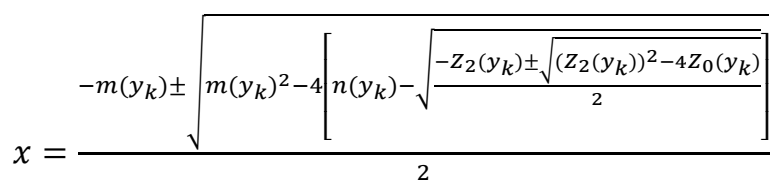

For $y_{k}=\omega_{k-1} \sqrt[5]{\frac{Y_{0}}{2}+\frac{1}{2} \sqrt{Y_{0}{ }^{2}+4\left(\frac{Y_{3}}{5}\right)^{5}}}+\omega_{n-(k-1)} \sqrt[5]{\frac{Y_{0}}{2}-\frac{1}{0} \sqrt{Y_{0}{ }^{2}+4\left(\frac{Y_{3}}{5}\right)^{5}}}$

Thus, any quintic can be solved by radicals, as was the main goal of this work.

\subsection{Solving the sextic}

For the sextic equation we start from the "Bring-Jerrard normal Sextic (BJSx)" equation (required), $e(x)=x^{6}+p x^{2}+q x+r=0$, which after obtained by Bring's method, we will apply on it a new 4th degree Tschirnhaus transformation, $g(x, y)=x^{4}+d x^{3}+$ $c x^{2}+b x+a-y=0$ (with the same modifications used before in the expressions of the Bring coefficients for the quintic) so as to eliminate the $2^{\text {nd }}, 4^{\text {th }}$ and $6^{\text {th }}$ coefficients at once from its resultant, $R_{1}(y)=y^{6}+\boldsymbol{Y}_{5} y^{5}+Y_{4} y^{4}+\boldsymbol{Y}_{3} y^{3}+Y_{2} y^{2}+\boldsymbol{Y}_{1} y+Y_{0}=0$. This will allow us to obtain the De Moivre Sextic: $D M_{1}(y)=y^{6}+Y_{4} y^{4}+Y_{2} y^{2}+Y_{0}=0$, in which we will make the change $y^{2}=w$ to simplify it to a cubic equation, $f(w)=w^{3}+Y_{4} w^{2}+$ $Y_{2} w+Y_{0}$, which we already know how to work it. The following flowchart gives the steps to follow to solve the sextic (somewhat "similar" to those followed for the quintic):

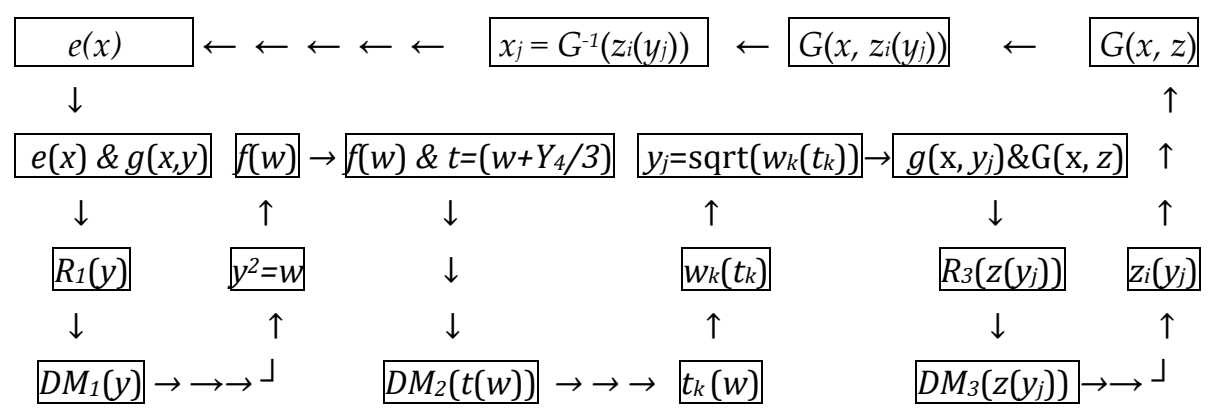

Figure 5. Flowchart of the steps to solve the Bring-Jerrard Normal Sextic Equation (BJS)

Making the coefficient $Y_{5}=0$, for $a=2 p / 3$, we obtain $e(x) \& g(x, y)$ : 


$$
\begin{aligned}
& \mathrm{cb}=\mathrm{x}^{\wedge} 6+\mathrm{p}^{*} \mathrm{x}^{\wedge} 2+\mathrm{q}^{*} \mathrm{x}+\mathrm{r} \\
& \text { res }=\text { cb.resultant }\left(-y+x^{\wedge} 4+d^{*} x^{\wedge} 3+c^{*} x^{\wedge} 2+b^{*} x+2^{*} p / 3, x\right) \cdot \operatorname{poly}(y) \\
& \text { Resultant equation is: } \quad R_{1}(y)=y^{6}+Y_{4} y^{4}+Y_{3} y^{3}+Y_{2} y^{2}+Y_{1} y+Y_{0}=0 \\
& Y_{4}=1 / 3\left(6 c^{2} p+12 b d p+15 c d q+9 d^{2} r-2 p^{2}+15 b q+18 c r\right) \\
& Y_{3}=-1 / 27\left(108 c d^{2} p^{2}+81 d^{3} p q-108 b^{2} c p+36 c^{2} p^{2}+72 b d p^{2}-135 b c^{2} q\right. \\
& -135 b^{2} d q+306 c d p q+135 d^{2} q^{2}-54 c^{3} r-324 b c d r+162 d^{2} p r \\
& \left.+4 p^{3}+63 b p q+135 c q^{2}-162 b^{2} r+54 c p r+297 d q r+54 r^{2}\right) \\
& Y_{2}=1 / 9\left(9 d^{4} p^{3}+9 c^{4} p^{2}-36 b c^{2} d p^{2}+18 b^{2} d^{2} p^{2}+36 c d^{2} p^{3}+9 c^{3} d p q-63 b c d^{2} p q\right. \\
& +63 d^{3} p^{2} q+45 c^{2} d^{2} q^{2}-45 b d^{3} q^{2}-81 c^{2} d^{2} p r+18 b d^{3} p r \\
& +36 c d^{3} q r+27 d^{4} r^{2}+9 b^{4} p-36 b^{2} c p^{2}-6 c^{2} p^{3}-12 b d p^{3} \\
& +45 b^{3} c q-63 b c^{2} p q+27 b^{2} d p q+57 c d p^{2} q-45 c^{3} q^{2}-45 b c d q^{2} \\
& +99 d^{2} p q^{2}+81 b^{2} c^{2} r+54 b^{3} d r+54 c^{3} p r-108 b c d p r+27 d^{2} p^{2} r \\
& -27 c^{2} d q r-162 b d^{2} q+p^{4}-15 b p^{2} q+45 b^{2} q^{2}+36 c p q^{2}+45 d q^{3} \\
& -27 b^{2} p r-18 c p^{2} r-27 b c q r+72 d p q r+81 c^{2} r^{2}-162 b d r^{2} \\
& \left.+36 q^{2} r\right) \\
& Y_{1}=-1 / 81\left(108 d^{4} p^{4}-81 b d^{4} p^{2} q+81 c d^{4} p q^{2}-81 d^{5} q^{3}-162 c d^{4} p^{2} r+243 d^{5} p q r\right. \\
& +108 c^{4} p^{3}-432 b c^{2} d p^{3}+216 b^{2} d^{2} p^{3}-81 b c^{4} p q+324 b^{2} c^{2} d p q \\
& -162 b^{3} d^{2} p q+108 c^{3} d p^{2} q-432 b c d^{2} p^{2} q+432 d^{3} p^{3} q+81 c^{5} q^{2} \\
& -405 b c^{3} d q^{2}+405 b^{2} c d^{2} q^{2}+216 c^{2} d^{2} p q^{2}-621 b d^{3} p q^{2} \\
& +405 c d^{3} q^{3}-162 c^{5} p r+648 b c^{3} d p r-324 c^{2} d^{2} p^{2} r+216 b d^{3} p^{2} r \\
& +81 c^{4} d q r-729 b c^{2} d^{2} q r+567 b^{2} d^{3} q r-702 c d^{3} p q r-81 d^{4} q^{2} r \\
& +486 c^{3} d^{2} r^{2}-972 b c d^{3} r^{2}+486 d^{4} p r^{2}+108 b^{4} p^{2}-24 c^{2} p^{4} \\
& -48 b d p^{4}-81 b^{5} q+216 b^{3} c p q-378 b c^{2} p^{2} q+540 b^{2} d p^{2} q \\
& -60 c d p^{3} q+405 b^{2} c^{2} q^{2}-405 b^{3} d q^{2}-378 c^{3} p q^{2}+27 b c d p q^{2} \\
& +648 d^{2} p^{2} q^{2}-405 c^{2} d q^{3}-405 b d^{2} q^{3}-486 b^{4} c r+540 c^{3} p^{2} r \\
& -648 b c d p^{2} r-36 d^{2} p^{3} r+324 b c^{3} q r+972 b^{2} c d q r+648 c^{2} d p q r \\
& -891 b d^{2} p q r+324 c d^{2} q^{2} r-486 c^{4} r^{2}+1458 b^{2} d^{2} r^{2}-486 c d^{2} p r^{2} \\
& -81 d^{3} q r^{2}-4 p^{5}-33 b p^{3} q+297 b^{2} p q^{2}-27 c p^{2} q^{2}+405 b c q^{3} \\
& +459 d p q^{3}-18 c p^{3} r-648 b^{3} q r-648 b c p q r \\
& -81 d p^{2} q r-162 c^{2} q^{2} r-729 b d q^{2} r+648 c^{2} p r^{2}-648 b d p r^{2} \\
& +648 c d q r^{2}-486 d^{2} r^{3}+81 q^{4}+108 p q^{2} r-54 p^{2} r^{2}-567 b q r^{2} \\
& \left.+486 c r^{3}\right)
\end{aligned}
$$

Let's leave it at that for now. The modified Bring variables to be applied to $Y_{3}$, since the expression of $a=2 p / 3$ was already introduced, will be: $c=d+h+k$ and $b=f d+h+j$. The result of these changes is factored over the variable $d$, obtaining: $Y_{3}=D_{3} d^{3}+D_{2} d^{2}+$ $D_{1} d+D_{0}$. Making, following Bring, the $D^{\prime} s$ equal to zero, $Y_{3}$ becomes eliminated. Next are some details realized in this process. For:

$$
c=d+h+k ; \quad b=f^{*} d+h+j
$$

expand $\left(-1 / 27^{*}\left(108^{*} c^{*} d^{\wedge} 2^{*} p^{\wedge} 2+81^{*} d^{\wedge} 3^{*} p^{*} q-108^{*} b^{\wedge} 2^{*} c^{*} p+36^{*} c^{\wedge} 2^{*} p^{\wedge} 2+72^{*} b^{*} d^{*} p^{\wedge} 2-\right.\right.$ $135^{*} b^{*} c^{\wedge} 2^{*} q-135^{*} b^{\wedge} 2^{*} d^{*} q+306^{*} c^{*} d^{*} p^{*} q+135^{*} d^{\wedge} 2^{*} q^{\wedge} 2-54^{*} c^{\wedge} 3^{*} r-324^{*} b^{*} c^{*} d^{*} r+$ $162^{*} \mathrm{~d}^{\wedge} 2^{*} \mathrm{p}^{*} \mathrm{r}+4^{*} \mathrm{p}^{\wedge} 3+63^{*} \mathrm{~b}^{*} \mathrm{p}^{*} \mathrm{q}+135^{*} \mathrm{c}^{*} \mathrm{q}^{\wedge} 2-16^{*} 2^{*} \mathrm{~b}^{\wedge} 2^{*} \mathrm{r}+54^{*} \mathrm{c}^{*} \mathrm{p}^{*} \mathrm{r}+297^{*} \mathrm{~d}^{*} \mathrm{q}^{*} \mathrm{r}+$ $\left.\left.54^{*} \mathrm{r}^{\wedge} 2\right)\right) \cdot \operatorname{poly}(\mathrm{d})$ 


$$
\begin{aligned}
& D_{3}=-\left(4 f^{2} p+\right.\left.5 f^{2} q-4 p^{2}+5 f q-3 p q+12 f r+2 r\right) \\
& D_{2}=-1 / 3\left(12 f^{2} h p\right.+12 f^{2} k p+24 f h p+24 f j p-8 f p^{2}-12 h p^{2}-12 k p^{2}+60 f h q \\
&+30 f j q+30 f k q+18 f^{2} r+36 f h r+36 f k r-4 p^{2}+15 h q \\
&\left.+15 j q-34 p q-15 q^{2}+54 h r+36 j r+18 k r-18 p r\right) \\
& D_{1}=1 / 3\left(24 f h^{2} p+24 f h j p+24 f h k p+24 f j k p+15 f h^{2} q+30 f h k q+15 f k^{2} q\right. \\
&+ 12 h^{2} p+24 h j p+12 j^{2} p-16 h p^{2}-8 j p^{2}-8 k p^{2}+45 h^{2} q \\
&+60 h j q+15 j^{2} q+30 h k q+30 j k q-7 f p q-34 h p q-34 k p q \\
&+36 f h r+54 h^{2} r+36 f j r+36 h j r+72 h k r+36 j k r+18 k^{2} r \\
&\left.-15 q^{2}-6 p r-33 q r\right) \\
& D_{0}=4 h^{3} p+8 h^{2} j p+4 h j^{2} p+4 h^{2} k p+8 h j p p+4 j^{2} k p-4 / 3 h^{2} p^{2}-8 / 3 h k p^{2} \\
&-4 / 3 k^{2} 2 p^{2}+5 h^{3} q+5 h^{2} j q+10 h^{2} k q+10 h j k q+5 h k^{2} q+5 j k^{2} q \\
&+ 2 h^{3} r+6 h^{2} k r+6 h k^{2} r+2 k^{3} r-3 / 3 k^{2} 2 p^{2}-7 / 3 h p q-7 / 3 j p q \\
&-5 h q^{2}-5 k q^{2}+6 h^{2} r+12 h j r+6 j^{2} r-2 h p r-2 k p r-2 r^{2}
\end{aligned}
$$

Making, $D_{3}=D_{2}=D_{1}=D_{1}=0$, the values of variables $f, \boldsymbol{j}, \boldsymbol{h}$ and $\boldsymbol{k}$ are obtained:

solve $(\mathrm{D} 3==0, \mathrm{f})$

$$
\begin{aligned}
f=-1 / 2(5 q+ & 12 r \pm \operatorname{sqrt}\left(64 p^{3}+128 p^{2} q+5(12 p+5) q^{2}-16(2 p-5 q) r\right. \\
+ & \left.\left.144 r^{2}\right)\right) /(4 p+5 q),
\end{aligned}
$$

And a constant value for the unknown $f$ is again obtained, as it turned out to depend only on the known constant coefficients $p, q$, and $r$. With $D_{2}=0$ we clear $j$ :

$$
\begin{aligned}
& \text { solve }(\mathrm{D} 2==0, \mathrm{j}) \\
& \begin{aligned}
j=1 / 3(4(2 f+ & 3 \boldsymbol{h}+3 \boldsymbol{k}+1) p^{2}-12\left(f^{2} \boldsymbol{k}+\left(f^{2}+2 f\right) \boldsymbol{h}\right) p-(15(4 f+1) \boldsymbol{h} \\
& +30 f \boldsymbol{k}-34 p) q+15 q^{2}-18\left(f^{2}+(2 f+3) \boldsymbol{h}+(2 f+1) \boldsymbol{k}\right. \\
- & p) r) /(8 f p+5(2 f+1) q+12 r)
\end{aligned}
\end{aligned}
$$

As can be seen, the value of $j$ becomes a function of $1^{\text {st }}$ degree of the unknowns $h$ and $k$, which is similar to what happened with the quintic with the variable $i$. Next we will calculate the roots $h_{i}$ of $D_{1}$, but with the calculated value of $j$ also included also in $D_{1}$.

solve $\left(1 / 3^{*}\left(24^{*} \mathrm{f}^{*} h^{\wedge} 2^{*} \mathrm{p}+24^{*} \mathrm{f}^{*} \mathrm{~h}^{*} \mathrm{j}^{*} \mathrm{p}+24^{*} \mathrm{f}^{*} \mathrm{~h}^{*} \mathrm{k}^{*} \mathrm{p}+24^{*} \mathrm{f}^{*} \mathrm{j}^{*} \mathrm{k}^{*} \mathrm{p}+15^{*} \mathrm{f}^{*} \mathrm{~h}^{\wedge} 2^{*} \mathrm{q}+30^{*} \mathrm{f}^{*} h^{*} \mathrm{k}^{*} \mathrm{q}+\right.\right.$ $15^{*} \mathrm{f}^{*} \mathrm{k}^{\wedge} 2^{*} \mathrm{q}+12^{*} \mathrm{~h}^{\wedge} 2^{*} \mathrm{p}+24^{*} \mathrm{~h}^{*} \mathrm{j}^{*} \mathrm{p}+12^{*} \mathrm{j}^{\wedge} 2^{*} \mathrm{p}-16^{*} \mathrm{~h}^{*} \mathrm{p}^{\wedge} 2-8^{*} \mathrm{j}^{*} \mathrm{p}^{\wedge} 2-8^{*} \mathrm{k}^{*} \mathrm{p}^{\wedge} 2+45^{*} \mathrm{~h}^{\wedge} 2^{*} \mathrm{q}+$ $60^{*} h^{*} j^{*} q+15^{*} j^{\wedge} 2^{*} q+30^{*} h^{*} k^{*} q+30^{*} j^{*} k^{*} q-7^{*} f^{*} p^{*} q-34^{*} h^{*} p^{*} q-34^{*} k^{*} p^{*} q+36^{*} f^{*} h^{*} r+$ $54^{*} h^{\wedge} 2^{*} r+36^{*} f^{*} j^{*} r+36^{*} h^{*} j^{*} r+72^{*} h^{*} k^{*} r+36^{* * *} k^{*} r+18^{*} k^{\wedge} 2^{*} r-15^{*} q^{\wedge} 2-6^{*} p^{*} r-$ $\left.\left.33^{*} \mathrm{q}^{*} \mathrm{r}\right)==0, \mathrm{~h}\right)$

$$
\begin{aligned}
h=-1 / 3(576 & f^{4} \boldsymbol{k} p^{3}-64(3 \boldsymbol{k}+1) p^{5}-64\left(4 f^{3}+6 f^{2} \boldsymbol{k}-3 f^{2}\right) p^{4} \\
& -25\left(15\left(4 f^{3}-3 f\right) \boldsymbol{k}-\left(56 f^{2}+56 f-17\right) p+12 p^{2}\right) q^{3}-375 q^{4} \\
& +1296\left(3 f^{2}+4 f \boldsymbol{k}+f-p\right) r^{3}+40\left(45 f^{2} \boldsymbol{k} p+\left(45 f^{2}+17 f\right.\right. \\
& \left.-15 \boldsymbol{k}) p^{2}-23 p^{3}\right) q^{2}-36\left(4(20 f+12 \boldsymbol{k}-3) p^{2}-12\left(8 f^{3}+f^{2}\right.\right. \\
+ & \left.\left(16 f^{2}-4 f-1\right) \boldsymbol{k}\right) p-15\left(4 f^{3}+10 f^{2}+\left(4 f^{2}+8 f+1\right) \boldsymbol{k}+f\right. \\
- & \left.2 p) q+30 q^{2}\right) r^{2}-16\left((15 \boldsymbol{k}+34) p^{4}-45\left(f^{4}+2 f^{3}\right) \boldsymbol{k} p^{2}+\left(20 f^{3}\right.\right. \\
- & \left.\left.34 f^{2}+30\left(f^{2}+f\right) \boldsymbol{k}-15 f\right) p^{3}\right) q-6\left(1 6 \left(16 f^{2}+6(2 f-1) \boldsymbol{k}\right.\right. \\
- & 6 f-1) p^{3}+16 p^{4}-48\left(3 f^{4}+2\left(6 f^{3}-f^{2}\right) \boldsymbol{k}\right) p^{2}-5\left(60 f^{3}\right.
\end{aligned}
$$




$$
\begin{aligned}
& \left.+45 f^{2}-15\left(4 f^{2}-8 f-1\right) k+(112 f-3) p\right) q^{2}+150 q^{3} \\
& +4\left(\left(50 f^{2}-18 f+60 k-39\right) p^{2}+15 p^{3}-15\left(3 f^{4}+6 f^{3}+2\left(4 f^{3}\right.\right.\right. \\
& \left.\left.\left.\left.+6 f^{2}-f\right) k\right) p\right) q\right)+\operatorname{sqr}\left(512\left(f^{2}+f-1\right) p^{7}+256 p^{8}+256\left(f^{4}\right.\right. \\
& \left.-6 f^{3}+3 f^{2}\right) p^{6}-5625(f-1) q^{6}+375\left(60 f^{3}-2(28 f-17) p\right. \\
& -45 f) q^{5}+25\left(\left(892 f^{2}+408 f+649\right) p^{2}-408 p^{3}+15\left(28 f^{4}\right.\right. \\
& \left.\left.-93 f^{2}\right) p\right) q^{4}+3888\left(3 f^{4}+18 f^{3}+3 f^{2}+2\left(3 f^{2}-7 f\right) p+3 p^{2}\right. \\
& -44 f q) r^{4}+40\left(\left(962 f^{2}+863 f\right) p^{3}-692 p^{4}-45\left(6 f^{4}+\right.\right. \\
& \left.\left.19 f^{3}\right) p^{2}\right) q^{3}+324\left(72 f^{4} p-8\left(22 f^{2}-4 f-1\right) p^{2}+8 p^{3}-\right. \\
& 5\left(32 f^{2}+148 f-12 p+11\right) q^{2}+2\left(135 f^{4}+60 f^{3}-\left(186 f^{2}-\right.\right. \\
& \left.\left.48 f-17) p+103 p^{2}\right) q\right) r^{3}+16\left((225 f-1096) p^{5}-\left(250 f^{3}-\right.\right. \\
& \left.\left.1726 f^{2}-435 f\right) p^{4}-45\left(7 f^{5}+26 f^{4}\right) f^{4}\right) q^{2}+18\left(6 4 \left(6 f^{2}-4 f-\right.\right. \\
& 3) p^{4}+192 p^{5}+192\left(f^{4}+2 f^{2}\right) p^{3}+75\left(8 f^{2}-136 f+12 p-\right. \\
& 17) q^{3}+450 q^{4}+30\left(45 f^{5}+45 f^{4}+(9 f+127) p^{2}-\left(74 f^{3}+\right.\right. \\
& \left.\left.220 f^{2}-79 f-7\right) p\right) q^{2}+8\left(135 f^{5} p-(113 f+204) p^{3}-\right. \\
& \left.\left.3\left(166 f^{3}-96 f^{2}-27 f\right) p^{2}\right) q\right) r^{2}-64\left(63 f^{5} p^{4}-(89 f-92) p^{6}+\right. \\
& \left.\left(46 f^{3}-72 f^{2}-15 f\right) p^{5}\right) q+3\left(1152 f^{4} p^{4}-128\left(22 f^{2}-4 f-\right.\right. \\
& 1) p^{5}+128 p^{6}+75\left(160 f^{3}+120 f^{2}-12(f-3) p-405 f-\right. \\
& 30) q^{4}+2250 q^{5}+20\left((1174 f+681) p^{2}+15\left(10 f^{3}-115 f^{2}+\right.\right. \\
& f) p) q^{3}-16\left(2\left(230 f^{2}-743 f+95\right) p^{3}+30 p^{4}+15\left(30 f^{4}+\right.\right. \\
& \left.\left.78 f^{3}-19 f^{2}\right) p^{2}\right) q^{2}-32\left(\left(210 f^{2}-24 f-29\right) p^{4}-115 p^{5}+\right. \\
& \left.\left.\left.\left.3\left(63 f^{4}-44 f^{3}\right) p^{3}\right) q\right) r\right)(8 f p+5(2 f+1) q+12 r)\right) /\left(192 f^{4} p^{3}-\right. \\
& 128 f^{2} p^{4}-64 p^{5}-125\left(4 f^{3}-3 f\right) q^{3}+1728 f r^{3}+200\left(3 f^{2} p-\right. \\
& \left.p^{2}\right) q^{2}+36\left(4\left(16 f^{2}-4 f-1\right) p-16 p^{2}+5\left(4 f^{2}+8 f+\right.\right. \\
& 1) q) r^{2}-80\left(2\left(f^{2}+f\right) p^{3}+p^{4}-3\left(f^{4}+2 f^{3}\right) p^{2}\right) q-6(32(2 f- \\
& 1) p^{3}-32\left(6 f^{3}-f^{2}\right) p^{2}+25\left(4 f^{2}-8 f-1\right) q^{2}-40\left(\left(4 f^{3}+\right.\right. \\
& \left.\left.\left.\left.6 f^{2}-f\right) p-2 p^{2}\right) q\right) r\right)
\end{aligned}
$$

Again, as with the quintic, the two roots $\boldsymbol{h}_{1,2}$ turn out to be also linearly dependent only on the variable $k$ as well, since the expression inside the square root depends only on the values $\boldsymbol{f}, \boldsymbol{p}, \boldsymbol{q}$, and $\boldsymbol{r}$. As seen, coefficients can have too long expressions and when using Sagemath on our memory limited laptop they sometimes do not work correctly. Therefore, we could use shorter expressions for them, if it were necessary. For example:

$$
\begin{aligned}
& \mathbf{h}_{\mathbf{i}}=\mathrm{H} 01+\mathrm{H} 10^{*} \mathrm{k} \pm \mathrm{H} 02 ; \text { but, if we put } \mathrm{H} 00=\mathrm{H} 01 \pm \mathrm{H} 02, \text { it simplifies to: }+ \\
& \mathbf{h}_{\mathbf{i}}=\mathbf{H} \mathbf{H 0 0}+\mathbf{H} 1 \mathbf{1 0}^{*} \mathbf{k}
\end{aligned}
$$

Introducing the chosen value $\boldsymbol{h}$ (with its original appearance, not simplified) in the expression of the variable $j$ to make it depend only on $k$, we have:

expand $\left(1 / 3^{*}\left(4^{*}\left(2^{*} \mathrm{f}+3^{*} \mathrm{~h}+3^{*} \mathrm{k}+1\right)^{*} \mathrm{p}^{\wedge} 2-12^{*}\left(\mathrm{f}^{\wedge} 2^{*} \mathrm{k}+\left(\mathrm{f}^{\wedge} 2+2^{*} \mathrm{f}\right)^{*} \mathrm{~h}\right)^{*} \mathrm{p}-\left(15^{*}\left(4^{*} \mathrm{f}+1\right)^{*} \mathrm{~h}+\right.\right.\right.$ $\left.\left.30^{*} \mathrm{f}^{*} \mathrm{k}-34^{*} \mathrm{p}\right)^{*} \mathrm{q}+15^{*} \mathrm{q}^{\wedge} 2-18^{*}\left(\mathrm{f}^{\wedge} 2+\left(2^{*} \mathrm{f}+3\right)^{*} \mathrm{~h}+\left(2^{*} \mathrm{f}+1\right)^{*} \mathrm{k}-\mathrm{p}\right)^{*} \mathrm{r}\right) /\left(8^{*} \mathrm{f}^{*} \mathrm{p}+5^{*}\left(2^{*} \mathrm{f}+1\right)^{*} \mathrm{q}+\right.$ $\left.\left.12^{*} \mathrm{r}\right)\right) \cdot \operatorname{poly}(\mathrm{k})$

Writing a shorter expression for $j$, it could be reduced to: $\mathbf{j}=\mathbf{J} 00+\mathbf{J} 0 \mathbf{1}^{*} \mathbf{k}$. Entering the values found for $h$ and $j$ in the coefficient $D_{0}$ so that it depends only on $k$. Let us call it D0K. Equaling D0K to zero, we obtain a long cubic equation in $k$, which will also be written in abbreviated form. Let's see, for example, taking: 
$\mathrm{j}=\mathrm{J} 00+\mathrm{J1}^{*} \mathrm{k}, \quad \mathrm{h}=\mathrm{H} 00+\mathrm{H} 01^{*} \mathrm{k}, \quad \mathrm{c}=\mathrm{d}+\mathrm{h}+\mathrm{k}, \quad \mathrm{b}=\mathrm{f}^{*} \mathrm{~d}+\mathrm{h}+\mathrm{j}$

And expressing the long result of D0K in another shorter form, we have:

$\mathrm{D} 0 \mathrm{~K}=\mathrm{K} 00+\mathrm{K} 01^{*} \mathrm{k}+\mathrm{K} 02^{*} \mathrm{k}^{\wedge} 2+\mathrm{K} 03^{*} \mathrm{k}^{\wedge} 3=0$

Thus, by solving this cubic equation we can have the numerical roots of D0K, $\boldsymbol{k}_{1}, \boldsymbol{k}_{2}$ and $\boldsymbol{k}_{3}$. Taking the $\boldsymbol{k}_{i}$ that returns the normal sextic equation, and introducing it in the variables $j$ and $h$ we get to know them all. Thus, we will have eliminated $Y_{3}$ and $Y_{5}$. Therefore, we only have to eliminate $Y_{1}$ to obtain: $D M_{1}(y)=y^{6}+Y_{4} y^{4}+Y_{2} y^{2}+Y_{0}=0$.

To eliminate the coefficient $Y_{1}$, we expand it with all the numerical values already known as constant values of $f, j, h$ and $\boldsymbol{k}$ introduced into the variables $\boldsymbol{c}=\boldsymbol{d}+\boldsymbol{h}+\boldsymbol{k}$ and $\boldsymbol{b}$ $=f d+h+j$. Thus, $Y_{1}$ would become depending only on the last unknown variable that remains, $d$. This gives rise to a quintic auxiliary equation in $d$, Y1D. This auxiliary quintic equation is solved according to the procedure described in section 3.3. And that root of the five ones of the Y1D equation that returns the original Bring sextic equation is chosen.

Thus, it was possible to eliminate in equation (29) the 2nd term $\left(Y_{5}\right)$, as well as the terms 4th $\left(Y_{3}\right)$ and 6th $\left(Y_{1}\right)$, and the obtained equation was: $D M_{1}(y)=y^{6}+Y_{4} y^{4}+Y_{2} y^{2}+$ $Y_{0}=0$, which was easily solvable because when making the change of variable, $w=y^{2}$, it became a cubic equation, $w^{3}+Y_{4} w^{2}+Y_{2} w+Y 0=0$. This fact simplified the solution of the sextic equation. That is, the requirements of satisfying the internal relation, $\left(\frac{Y_{4}}{6}\right)^{2}=\frac{Y_{2}}{9}$, to complete the De Moivre sextic form, which involved a new equation and a new variable to be created, became unnecessary. We only needed to know the three roots of the reduced cubic equation, constructed according to the following process:

$$
y^{6}+Y_{4} y^{4}+Y_{2} y^{2}+Y_{0}=0 \stackrel{y^{2}=w}{\longrightarrow} w^{3}+Y_{4} w^{2}+Y_{2} w+Y 0=0,
$$

Changing again to a new variable $t=w+Y_{4} / 3$ to obtain the De Moivre cubic, $\boldsymbol{t}^{3}+$ $T_{1} t+T_{0}=0$, where $T_{1}=Y_{2}-\frac{1}{3}\left(Y_{4}\right)^{2}$ and $T_{0}=\frac{2}{27}\left(Y_{4}\right)^{3}-\frac{1}{3}\left(Y_{4}\right) Y_{2}+Y_{0}$ we can compare, $t^{3}+\boldsymbol{T}_{1} t+\boldsymbol{T}_{\mathbf{0}}=0$, for $t=u+v$, with the cubic binomial, $(u+v)^{3}-3 u v(u+v)-$ $\left(u^{3}+v^{3}\right)=0$. From this, $-u v=\frac{T_{1}}{3}$, and $-\left(u^{3}+v^{3}\right)=-\beta_{3}=\boldsymbol{T}_{\mathbf{0}}$. which allows us to calculate, $u$ and $v$; and then: $t=u+v ; w=-\frac{Y_{4}}{3}+t ; y=\sqrt{w}$.

$$
\begin{aligned}
& v=-\frac{\boldsymbol{T}_{\mathbf{1}}}{3 u} ; \quad \boldsymbol{T}_{\mathbf{0}}=-\left(u^{3}+v^{3}\right) \rightarrow u^{3}-\left(\frac{\boldsymbol{T}_{\mathbf{1}}}{3 u}\right)^{3}+\boldsymbol{T}_{\mathbf{0}}=0 \\
& u^{6}+\boldsymbol{T}_{\mathbf{0}} u^{3}-\frac{1}{27} \boldsymbol{T}_{\mathbf{1}}{ }^{3}=0 \rightarrow\left(u^{3}\right)^{2}+\boldsymbol{T}_{\mathbf{0}}\left(u^{3}\right)-\frac{1}{27} \boldsymbol{T}_{\mathbf{1}}{ }^{3}=0 \\
& u=\sqrt[3]{\frac{-T_{0}+\sqrt{T_{0}^{2}+4\left(\frac{T_{1}}{3}\right)^{3}}}{2}} ; \quad v=\sqrt[3]{\frac{-T_{0}-\sqrt{T_{0}^{2}+4\left(\frac{T_{1}}{3}\right)^{3}}}{2}}, \\
& t_{k}=\omega_{k-1} u+\omega_{3-(k-1)} v, \text { for } \begin{array}{ll}
t_{1} & =\omega_{0} u+\omega_{3} v \\
t_{2} & =\omega_{1} u+\omega_{2} v \\
t_{3} & =\omega_{2} u+\omega_{1} v
\end{array} \quad \text { where, } \quad \begin{array}{c}
\omega_{m}=e^{e^{k\left(m \frac{2 \pi}{3}\right)}} \\
\text { for } k=1,2,3 \\
m=0, \ldots, 3
\end{array}
\end{aligned}
$$

Undoing the change, $w=-\frac{Y_{4}}{3}+t$, for $t=u+v$ the basic cubic solution arises, to construct the others, based on the roots of unit $\omega_{m}=e^{j\left(m \frac{2 \pi}{3}\right)}, k=1,2,3$ : 
$w_{k}=-\frac{Y_{4}}{3}+t_{k}=-\frac{Y_{4}}{3}+\omega_{k-1} \sqrt[3]{\frac{-T_{0}+\sqrt{T_{0}{ }^{2}+4\left(\frac{T_{1}}{3}\right)^{3}}}{2}}+\omega_{3-(k-1)} \sqrt[3]{\frac{-T_{0}-\sqrt{T_{0}{ }^{2}+4\left(\frac{T_{1}}{3}\right)^{3}}}{2}}$

So, the roots of the equation, $y^{6}+Y_{4} y^{4}+Y_{2} y^{2}+Y_{0}=0$, for $y=\sqrt{w}$, are:

$$
\begin{gathered}
y_{k, 3+k}= \pm \sqrt{-\frac{Y_{4}}{3}+\omega_{k-1} \sqrt[3]{\frac{-T_{0}+\sqrt{T_{0}{ }^{2}+4\left(\frac{T_{1}}{3}\right)^{3}}}{2}}+\omega_{4-k} \sqrt[3]{\frac{-T_{0}-\sqrt{T_{0}{ }^{2}+4\left(\frac{T_{1}}{3}\right)^{3}}}{2}}} \\
y_{1,4}= \pm \sqrt{-\frac{Y_{4}}{3}+\omega_{0} u+\omega_{3} v} ; y_{2,5}= \pm \sqrt{-\frac{Y_{4}}{3}+\omega_{1} u+\omega_{2} v} ; y_{3,5}= \pm \sqrt{-\frac{Y_{4}}{3}+\omega_{2} u+\omega_{1} v}
\end{gathered}
$$

On the other hand, to undo the Tschirnhaus transformation of the fourth degree, for returning from the $y_{k, 3+k}$ to the Bring's sextic equation, the numerical values of $d, c, b, a$, and those of the six roots $y_{k, 3+k}$ are substituted into the $g(x)=x^{4}+d x^{3}+c x^{2}+b x+$ $a-y_{k, 3+k}=0$. This quartic is then transformed into a De Moivre's quartic form through a $2^{\text {nd }}$ degree transformation, $G(x, z)=x^{2}+m x+n-z=0$. This equation is solved and two roots $x_{j}$ are obtained, depending on the four roots $z_{i}$ of the De Moivre quartic resultant as a function of $m$ and $n$. Of these, only one return the Bring-Jerrard Sextic equation (BJS), $x^{6}+p x^{2}+q x+r=0$, which is taken. See:

$$
\begin{array}{lr}
g(x)=x^{4}+d x^{3}+c x^{2}+b x+a-y_{k, 3+k}=0 \& G(x, z)=x^{2}+m x+n-z=0 \downarrow & \leftarrow x=\frac{-m \pm \sqrt{m^{2}-4(n-\mathbf{z})}}{2} \\
\rightarrow \operatorname{Res}_{3}[g(x) \& G(x)]=z^{4}+Z_{3} z^{3}+Z_{2} z^{2}+Z_{1} z+Z_{0}=0 \stackrel{Z_{3}=Z_{1}=0}{\longrightarrow} z^{4}+Z_{2} z^{2}+Z_{0}=0 \rightarrow \boldsymbol{z}= \pm \sqrt{\frac{-Z_{2} \pm \sqrt{\left(Z_{2}\right)^{2}-4\left(Z_{0}\right)}}{2}} \uparrow
\end{array}
$$

Figure 6. Flowchart of the steps to undo the Tschirnhaus transformation of the $4^{\text {th }}$ degree for solving the sextic.

Calculations of the expression of $n$ by making $Z_{3}$ equal to zero $\left(n=-d^{2}+d m+\right.$ $2 c / 4$ ), of $m$ by making $Z_{1}$ equal to zero (which creates a cubic equation), and of $Z_{2}$ and of $Z_{0}$, can be observed in the process carried out for obtaining the resultant of $g(x) \& G(x, z)$ in order to obtain equations similar to (26), (27), and (28), which are almost exactly the same as those used now to obtain the resultant of $g(x) \& G(x, z)$ for the sextic.

Thus, $z_{i}=\sqrt{\frac{-z_{2} \pm \sqrt{\left(Z_{2}\right)^{2}-4\left(Z_{0}\right)}}{2}}$. But, from $x^{2}+m x+n-z=0 \rightarrow x=\frac{-m \pm \sqrt{m^{2}-4(n-z)}}{2}$, and then, we get the expression of the $x_{k, 3+k}$, where, as indicated, the values of $Z_{2}$ and $Z_{0}$ are constructed based on the values of $y=y_{k, 3+k}$, calculated in equation (31). For single values of $z_{i}\left(y_{k, 3+k}\right), m\left(y_{k, 3+k}\right)$ and $n\left(y_{k, 3+k}\right)$, the expression of $x=x_{k, 3+k}$ becomes:

$x_{k, 3+k}=\frac{-m\left(y_{k, 3+k}\right) \pm \sqrt{\left(m\left(y_{k, 3+k}\right)\right)^{2}-4\left[n\left(y_{k, 3+k}\right)-\sqrt{\frac{-z_{2}\left(y_{k, 3+k}\right) \pm \sqrt{\left(Z_{2}\left(y_{k, 3}+k\right)\right)^{2}-4 Z_{0}\left(y_{k, 3}+k\right)}}{2}}\right]}}{2}$

\subsection{Trying to solve the septic}

Starting from the normal septic equation (BJSp), $d(z)=z^{7}+Z_{3} z^{3}+Z_{2} z^{2}+Z_{1} z+$ $Z_{0}=0$, without the $2^{\text {nd }}, 3^{\text {rd }}$, and $4^{\text {th }}$ term, applying a strategy similar to that in the quintic it is necessary in a first step to obtain the bi-normal expression, $e(x)=x^{7}+X_{2} x^{2}+X_{1} x+$ $X_{0}=0$, (without the $2^{\text {nd }}, 3^{\text {rd }}, 4^{\text {th }}$ and $5^{\text {th }}$ terms). This expression would be required because we would need, in a second step, to form four equations for constructing the De Moivre septic (DMSp) $f(y)=y^{7}+Y_{5} y^{5}+Y_{3} y^{3}+Y_{1} y+Y_{0}=0$, to compare it with the binary identity $y^{7}-7 \alpha y^{5}+14 \alpha^{2} y^{3}-7 \alpha^{3} y-\beta_{7}=0$. Namely, three equations for elim- 
inating the $2^{\text {nd }}, 4^{\text {th }}$ and $6^{\text {th }}$ terms and the last equation to satisfy the internal condition, $\left(Y_{5} / 7\right)^{3}-2\left(Y_{5} / 7\right)\left(Y_{3} / 14\right)^{2}+\left(Y_{1} / 7\right)=(\alpha)^{3}-2(\alpha)\left(\alpha^{2}\right)+\alpha^{3}=0$. This expression would relate the three coefficients, in function of the last unknown $d$. After obtaining $d$, we would determine $\alpha=u v$ and $u^{7}+v^{7}$, and we would have the DMSp to solve it by radicals.

But, as it is known, so far it has not been possible to eliminate four terms of a general monic equation of degree less than 10 [7]. We have succeeded, however, in eliminating four terms at once from the general monic equation of degree 7 , or septic, and this is our only contribution to this problem in this paper. It was not possible to eliminate the three inter-sequential terms (2nd, 4 th and 6th) of the septic. That is, we have only developed the first step of the problem, and we have decided to leave the septic problem up to this point. This explains the reason for the title of this section. The steps, using Sagemath without the calculation details (more than two hundred pages of this type), are:

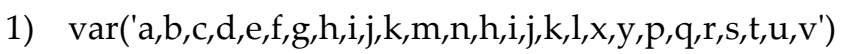

$$
\begin{aligned}
& \text { cb }=x^{\wedge} 7+p^{*} x^{\wedge} 3+q^{*} x^{\wedge} 2+r^{*} x+s \\
& \text { res }=\text { cb.resultant }\left(-y+x^{\wedge} 6+f^{*} x^{\wedge} 5+e^{*} x^{\wedge} 4+d^{*} x^{\wedge} 3+c^{*} x^{\wedge} 2+b^{*} x+\left(4^{*} e^{*} p+5^{*} f^{*} q+\right.\right. \\
& \left.\left.6^{*} r\right) / 7, x\right) \cdot \operatorname{poly}(y)
\end{aligned}
$$

from this result we obtained the resultant:

$$
f(y)=y^{7}+Y_{5} y^{5}+Y_{4} y^{4}+Y_{3} y^{3}+Y_{2} y^{2}+Y_{1} y+Y_{0}=0
$$

Using specially related coefficients to avoid auxiliary equations of degree $>6$, as:

$$
\begin{aligned}
& e=f+g \\
& d=m^{*} f+g \\
& c=f+h+j+k \\
& b=t^{*} f+h+i+j+k
\end{aligned}
$$

2) and expanding $Y_{5}$, a first part of coefficients of the variable $f$ are obtained:

$$
Y_{5}=\mathrm{F} 12 \cdot f^{2}+\mathrm{F} 11 . f+\mathrm{F} 10
$$

3) By expanding $Y_{4}$ the second part of coefficients of the variable $f$ arise:

$$
Y_{4}=\mathrm{F} 23 \cdot f^{3}+\mathrm{F} 22 \cdot f^{2}+\mathrm{F} 21 \cdot f+\mathrm{F} 20
$$

Making the coefficients of the variable $f$ equal to zero, $Y 5$ and $Y 6$ become eliminated. We obtained for the variables $t, m, h, i, j, k$ and $g$ the following results and interpretations:

solve $(\mathrm{F} 12==0, \mathrm{t})$

$t=$ Results in a linear function of only the variable $m$

solve $(\mathrm{F} 23==0, \mathrm{~m})$

It results in an equation of $5^{\text {th }}$ degree (depending only on the variable $m$ ) that we know how to solve according to section 3.3. So, $t$ and $m$ depend only on the constants $p, q, r, s$ and will be treated as numbers.

solve(F11 $=0, \mathrm{~h}$ )

$h=$ Results in a linear function of $g, i, j, k$.

solve(F22 $=0, \mathrm{~g})$

$g=$ Results in a linear function dependent only on $i$. 
Entering the expression of $g$ in $h$ we obtain $h$ as a linear function of $i, j, k$. And, introducing again the expressions of $g$ and $h$ in the expression of F10 and solving, we have:

solve(F10 $=0, \mathrm{i})$

$i=$ Results in a quadratic expression depending only on the number $m$ (and on $p, q$,

$r, s)$, thus it will be treated also as a number. Let the valid root be $i_{0}$ and $g_{0}$ as well.

Entering the expressions of $i_{0}, g_{0}$ and $h$, in F21 is obtained a long expression, depending on the variables $j$ and $k$, that can be simplified as:

$$
\mathrm{F} 21=\mathrm{a} 0 \cdot j^{2}+(\mathrm{b} 1 \cdot k+\mathrm{b} 0) \cdot j+\left(\mathrm{c} 2 \cdot k^{2}+\mathrm{c} 1 \cdot k+\mathrm{c} 0\right),
$$

where a0, b1, b0, c2,c1, c0 are numbers. Using Sagemath for solving it, we have:

$$
\begin{aligned}
& \text { solve }\left(\mathrm{a} 0^{*} \mathrm{j}^{\wedge} 2+\left(\mathrm{b} 1^{*} \mathrm{k}+\mathrm{b} 0\right)^{*} \mathrm{j}+\left(\mathrm{c} 2^{*} \mathrm{k}^{\wedge} 2+\mathrm{c} 1^{*} \mathrm{k}+\mathrm{c} 0\right)==0, \mathrm{j}\right) \\
& {\left[\mathrm{j}=-1 / 2^{*}\left(\mathrm{~b} 1^{*} \mathrm{k}+\mathrm{b} 0 \pm \operatorname{sqrt}\left(\left(\mathrm{b} 1^{\wedge} 2-4^{*} \mathrm{a} 0^{*} \mathrm{c} 2\right)^{*} \mathrm{k}^{\wedge} 2+\mathrm{b} 0^{\wedge} 2-4^{*} \mathrm{a} 0^{*} \mathrm{c} 0+2^{*}\left(\mathrm{~b} 0^{*} \mathrm{~b} 1-\right.\right.\right.\right.}
\end{aligned}
$$
$\left.\left.\left.2^{*} \mathrm{a} 0 * \mathrm{c} 1\right)^{*} \mathrm{k}\right)\right) / \mathrm{a} 0$,

$\mathrm{j}==-1 / 2^{*}\left(\mathrm{~b} 1^{*} \mathrm{k}+\mathrm{b} 0-\operatorname{sqrt}\left(\left(\mathrm{b} 1^{\wedge} 2-4^{*} \mathrm{a} 0^{*} \mathrm{c} 2\right)^{*} \mathrm{k}^{\wedge} 2+\mathrm{b} 0^{\wedge} 2-4^{*} \mathrm{a} 0^{*} \mathrm{c} 0+2^{*}\left(\mathrm{~b} 0^{*} \mathrm{~b} 1-\right.\right.\right.$ $\left.\left.\left.2^{*} \mathrm{a} 0^{*} \mathrm{c} 1\right)^{*} \mathrm{k}\right)\right) / \mathrm{a} 0$ ]

Introducing, after checking, the correct root $j$ in F20, let's suppose it is that of minus sign, then it is obtained another long expression that can be, as above, simplified to:

$$
\begin{aligned}
& \mathrm{j}=-1 / 2\left(\mathrm{~b} 1^{*} \mathrm{k}+\mathrm{b} 0-\mathrm{SQ}\right) \mathrm{a} 0 \\
& \mathrm{i}=\mathrm{i} 0 \\
& \mathrm{~h}=\mathrm{d} 1^{*} \mathrm{j}+\mathrm{d} 2^{*} \mathrm{k}+\mathrm{d} 0 \\
& \mathrm{~g}=\mathrm{g} 0
\end{aligned}
$$

$\left.\mathrm{SQ}=\operatorname{sqrt}\left(\left(\mathrm{b} 1{ }^{\wedge} 2-4^{*} \mathrm{a} 0^{*} \mathrm{c} 2\right)^{*} \mathrm{k}^{\wedge} 2+\mathrm{b} 0^{\wedge} 2-4^{*} \mathrm{a} 0^{*} \mathrm{c} 0+2^{*}\left(\mathrm{~b} 0^{*} \mathrm{~b} 1-2^{*} \mathrm{a} 0^{*} \mathrm{c} 1\right)^{*} \mathrm{k}\right)\right) / \mathrm{a} 0=\operatorname{sqrt}\left(\mathbf{L} 2^{*} \mathbf{k}^{\wedge} 2+\mathbf{L} 1^{*} \mathbf{k}+\mathbf{L} 0\right)$

Applying $\mathrm{SQ}^{3}=\mathrm{SQ} . \mathrm{SQ}^{2}$, to leave only coefficients in SQ and coefficients in the variable $k$, so that after separating them, putting them on both sides of the equal sign and squaring them, we can obtain a six-degree auxiliary equation. See the steps:

$$
\begin{gathered}
\mathrm{M}^{*} \mathrm{k}^{\wedge} 3+\mathrm{M} 2^{*} \mathrm{k}^{\wedge} 2+\mathrm{M} 1^{*} \mathrm{k}+\mathrm{M} 0-\left(\mathrm{N} 2^{*} \mathrm{k}^{\wedge} 2+\mathrm{N} 1^{*} \mathrm{k}+\mathrm{N} 0\right)^{*} \mathrm{sqrt}\left(\mathrm{L} 2^{*} \mathrm{k}^{\wedge} 2+\mathrm{L} 1^{*} \mathrm{k}+\mathrm{L} 0\right)=0 \\
\left(\mathrm{M} 3^{*} \mathrm{k}^{\wedge} 3+\mathrm{M} 2^{*} \mathrm{k}^{\wedge} 2+\mathrm{M} 1^{*} \mathrm{k}+\mathrm{M} 0\right)^{\wedge} 2=\left(\left(\mathrm{N} 2^{*} \mathrm{k}^{\wedge} 2+\mathrm{N} 1^{*} \mathrm{k}+\mathrm{N} 0\right)^{*} \mathrm{sqrt}\left(\mathrm{L} 2^{*} \mathrm{k}^{\wedge} 2+\mathrm{L} 1^{*} \mathrm{k}+\mathrm{L} 0\right)\right)^{\wedge} 2 \\
\text { solve }\left(\left(\mathrm{M} 3^{*} \mathrm{k}^{\wedge} 3+\mathrm{M} 2^{*} \mathrm{k}^{\wedge} 2+\mathrm{M} 1^{*} \mathrm{k}+\mathrm{M} 0\right)^{\wedge} 2-\left(\left(\mathrm{N} 2^{*} \mathrm{k}^{\wedge} 2+\mathrm{N} 1^{*} \mathrm{k}+\mathrm{N} 0\right)^{*} \mathrm{sqrt}\left(\mathrm{L} 2^{*} \mathrm{k}^{\wedge} 2+\mathrm{L} 1^{*} \mathrm{k}+\mathrm{L} 0\right)\right)^{\wedge} 2=0, \mathrm{k}\right) \\
{\left[0=\left(\mathrm{L} 2^{*} \mathrm{~N} 2^{\wedge} 2-\mathrm{M} 3^{\wedge} 2\right)^{*} \mathrm{k}^{\wedge} 6+\left(2^{*} \mathrm{~L} 2^{*} \mathrm{~N} 1^{*} \mathrm{~N} 2+\mathrm{L} 1^{*} \mathrm{~N} 2^{\wedge} 2-2^{*} \mathrm{M} 2^{*} \mathrm{M} 3\right)^{*} \mathrm{k}^{\wedge} 5+\left(2^{*} \mathrm{~L} 1^{*} \mathrm{~N} 1^{*} \mathrm{~N} 2+\mathrm{L} 0^{*} \mathrm{~N} 2^{\wedge} 2+\right.\right.} \\
\left.\left(\mathrm{N} 1^{\wedge} 2+2^{*} \mathrm{~N} 0^{*} \mathrm{~N} 2\right)^{*} \mathrm{~L} 2-\mathrm{M} 2^{\wedge} 2-2^{*} \mathrm{M} 1^{*} \mathrm{M} 3\right)^{*} \mathrm{k}^{\wedge} 4+\left(2^{*} \mathrm{~L} 2^{*} \mathrm{~N} 0^{*} \mathrm{~N} 1+2^{*} \mathrm{~L} 0^{*} \mathrm{~N} 1^{*} \mathrm{~N} 2+\left(\mathrm{N} 1^{\wedge} 2+2^{*} \mathrm{~N} 0^{*} \mathrm{~N} 2\right)^{*} \mathrm{~L} 1-\right. \\
\left.2^{*} \mathrm{M} 1^{*} \mathrm{M} 2-2^{*} \mathrm{M} 0^{*} \mathrm{M} 3\right)^{*} \mathrm{k}^{\wedge} 3+\mathrm{L} 0^{*} \mathrm{~N} 0^{\wedge} 2+\left(\mathrm{L} 2^{*} \mathrm{~N} 0^{\wedge} 2+2^{*} \mathrm{~L} 1^{*} \mathrm{~N} 0^{*} \mathrm{~N} 1+\left(\mathrm{N} 1 \wedge 2+2^{*} \mathrm{~N} 0^{*} \mathrm{~N} 2\right)^{*} \mathrm{~L} 0-\mathrm{M} 1 \wedge 2-\right. \\
\left.\left.2^{*} \mathrm{M} 0^{*} \mathrm{M} 2\right)^{*} \mathrm{k}^{\wedge} 2-\mathrm{M} 0^{\wedge} 2+\left(\mathrm{L} 1^{*} \mathrm{~N} 0^{\wedge} 2+2^{*} \mathrm{~L} 0^{*} \mathrm{~N} 0^{*} \mathrm{~N} 1-2^{*} \mathrm{M} 0^{*} \mathrm{M} 1\right)^{*} \mathrm{k}\right]
\end{gathered}
$$

Thus, the resulting previous equation can be simplified to a sixth-degree equation,

$$
\mathrm{K} 6^{*} \mathrm{k}^{\wedge} 6+\mathrm{K} 5^{*} \mathrm{k}^{\wedge} 5+\mathrm{K} 4^{*} \mathrm{k}^{\wedge} 4+\mathrm{K} 3^{*} \mathrm{k}^{\wedge} 3+\mathrm{K} 2^{*} \mathrm{k}^{\wedge} 2+\mathrm{K} 1^{*} \mathrm{k}+\mathrm{K} 0=0
$$

which, according to the previous section 3.4, we know how to resolve.

Entering, after checking, the valid value of $k$ as a number in $j$, we know $j$. Entering $k$ and $j$ in $h$ we know $h$. In this way, all the variables are numbers, and so, we will have eliminated $\mathrm{Y} 6, \mathrm{Y} 5$ and $\mathrm{Y} 4$. Substituting all the variables with their numerical value in $\mathrm{Y} 3$ and equaling it to zero, an auxiliar equation of the $4^{\text {th }}$ degree results and the value of $f$ (the only unknown variable) becomes known. In this way, four terms become eliminated, which was really the main goal of this section. As with the quintic and sexticAll these 
results were also checked with numerical coefficients and approximate solutions for $g, i$, $j, k$.

\section{Final Comments and Conclusions}

The modified expressions chosen for the Bring coefficients, within the fourth degree Tschirnhaus transformation (4dTschT), applied to the Bring-Jerrard normal form (BJQ), allowed us to transform it into a De Moivre quintic equation (DMQ), where the variables $g$ and $h$, become linearly dependent on the variable $i$ within a second-degree expression. Similarly, when we worked the sextic, with the same transformation, $j$ and $h$ also became linearly dependent on the variable $k$. This convenient feature made the degrees of the resulting auxiliary equations $(1 \times 3)$ less than 5 for the quintic and $(n-1=5)$ less than 6 for the sextic, which led us to obtain radical solutions for both types of equations.

It is important to remember that one of the contributions of E. S. Bring in 1786 to mathematics was to take out three terms from the general quintic equation using an original $4^{\text {th }}$ degree transformation with a particular and successful relation among coefficients. For centuries this was impossible, because of the use of a $3 \mathrm{dTschT}$ on the quintic that always it took to an auxiliar equation of a degree $2 \times 3=6>5$. May be Bring was trying, really, to solve the quintic.

In the same line of reasoning, we tried to transform the BJQ into the DMQ, successfully, to solve it by radicals, through another $4 \mathrm{dTschT}$ using different Bring's coefficients related among them. The same trick worked for the Bring-Jerrard normal form of the sextic (BJSx), $x^{6}+p x^{2}+q x+r=0$, to transform it into an equation without the $2^{\text {nd }}$, $4^{\text {th }}$ and $6^{\text {th }}$ terms, which was transformed into a cubic, solvable by radicals. Namely, it was not necessary to satisfy the internal conditions of the DMSx, simplifying its solution.

And, applying again the curious Bring's trick, through a $6 \mathrm{dTschT}$ to the BringJerrard normal form of the septic (BJSp), $z^{7}+Z_{3} z^{3}+Z_{2} z^{2}+Z_{1} z+Z_{0}=0$, choosing Bring's coefficients successfully related among them, we could take out at once four terms $\left(2^{\text {nd }}, 3^{\text {rd }}, 4^{\text {th }}\right.$ and $\left.5^{\text {th }}\right)$ from the resultant and arrived at its binormal form, $x^{7}+$ $X_{2} x^{2}+X_{1} x+X_{0}=0$.

Although it was not possible by now to solve the septic equation by radicals in this work, we achieved the important step of the elimination of four terms at once in this equation, which can be considered as a first part of its solution by radicals, if this were possible. Notice that until this work it had not been possible the elimination of four terms at once from a seven-degree equation.

G. B. Jerrard indicated (1832), after Tschirnhaus (1683), that his method opened the possibility of eliminating at once four and more terms from $n$-degree equations [6]. However, according to Lord William R. Hamilton's review of his article, "It results, then, from this discussion, that the process described in the present article will not in general avail to take away four terms at once, from equations lower than the tenth degree" [7].

On other side, the flowcharts presented in each case suggest that the choice of valid values can be easily programmed by making simultaneous calculations and checking all possible results. This is somewhat cumbersome and in addition has the requirement of enough memory on the computer or laptop used to run all the valid and invalid results.

We also managed to detect that for the case of the general equation of degree $n$, even, it is necessary to eliminate $n / 2$ inter-sequential terms (2nd, 4 th, 6 th, ...) at once and thus, the resultant can be transformed into an equation of degree $n / 2$, making the change, $z=y^{2}$. If these actions were possible and the equation of degree $n / 2$ were solvable by radicals, it is not necessary to fulfill the internal relations of the De Moivre form of even degree $n$, but only an equation of degree $n-1$ is required to be solved.

The solutions of the general quintic and general sextic equations by radicals obtained in this work seem to contradict the theorems of Galois (1832) [8][5], Abel (1828) [5] and Ruffini (1799) [5] on the impossibility of solving general monic equations of degree $n \geq 5$, by radicals. The reasons for achieving the solution of the quintic in this work was that, following a procedure similar to Bring's, we were able to achieve the linear dependence between roots of second-degree equations and the other variables of the 
type $\boldsymbol{h}_{i}=\mathrm{H}_{01}+\mathrm{H}_{10} \boldsymbol{k} \pm \mathrm{H}_{02}=\mathrm{H}_{10} \boldsymbol{k}+\mathrm{H}_{00}$. We think, this type of possibility was not contemplated in the theorems proposed by these mathematicians.

The results achieved in this work, against the Ruffini, Abel and Galois theorems, would imply that it is necessary to further explore the possibility of solving equations of more than six degrees by radicals, by Bring transformations or of a new type. For this purpose, we suggest among others the readings: [4-10].

Funding: This research received no external funding.

Acknowledgements. I thank Prof. José G. Quintero M., for his helpful suggestions and recommendations, Prof. J. Conesa M., for his mathematical support and time dedicated to the review, Prof. Andrés Echezuría Q., for his help with the Sagemath routines, Prof. E. Tescari James for his proofreading work (all of them retirees, like me), and finally Nora Franco, Noreli Franco and Nadia Franco for their invaluable help in writing, and their encouragement and solidarity in and during the conduct of this research. In addition, this work has been carried out thanks to the useful Sagemath software and to the resources provided by the Department of Mathematics of the Universidad Autónoma de Madrid, for the on-line calculations. The use of DeepL Translator was also a great help to check and correct the English.

\section{References}

[1] Alexander Chen, Yang-Hui He, John McKay. Translation of Erland Samuel Bring's "Transformation of Algebraic Equations". 1786. arXiv:1711.09253v1 [math. HO] 25 Nov 2017; pp. 1-21

[2] Victor Adamchik, D. D. Jeffrey. "Polynomial Transformations on Tschirnhaus, Bring and Jerrard", ACM SIGSAM Bulletin, Volume 37, No. 3, Sept. 2003; pp. 90-94.

http://www.apmaths.uwo,c'a/ djeffrey/Offprints/Adamchik.pdf

[3] R. F. Green. Ehrenfried Walter von Tschirnhaus. 1683. "A method for removing all intermediate terms from a given equation". ACM SIGSAM Bulletin, Vol 37, No. 1, March 2003. English Translation; pp. 1-3

[4] Titus Piezas. "Solvable Brioschi Quintics, other One-Parameter Forms, and the j-function". April 22, 2006; pp.1-9

[5] Jacqueline Stedall. "From Cardano's great art to Lagrange's reflections: filling a gap in the history of algebra". The Queen's College Oxford, OX1 4AW United Kingdom. 2010.

[6] G. B. Jerrard, "An essay on the resolution of equations", Taylor and Francis, 1859; pp. 1-84

[7] William. R. Hamilton, "Inquiry into the validity of a method recently proposed by George B. Jerrard, Esq., for transforming and resolving equations of elevated degrees", British Association Report, Bristol 1836. Edited by David R. Wilkins. 2000; pp. 295-348

[8] Leonard E. Dickson, "Modern Algebraic Theories". Chapter X: Equations solvable by Radicals. Benj. H. Sanborn \& Co. Chicago. 1926; pp. 178-203

[9] L. Bruce King, "Beyond the Quartic Equation". Chapter 5: Algebraic Equations Soluble by Radicals, Chapter 6: The Kiepert Algorithm for Roots of the the General Quintic Equation , Chapter 7: The Methods of Hermite and Gordan for solving the General Quintic Equation, Chapter 8: Beyond the Quintic Equation. Birkhäuser. Boston. 1996; pp. 82-147

[10] Tomas Johansson, “Analytic Solutions to Algebraic Equations”, Thesis Report. ISSN 0348-2960. Linköping. 06-12-1998; pp. 1-58 